\title{
Design and Control of a Deformable Trees-Pruning Aerial Robot
}

\author{
Changliang Xu $\mathbb{D},,^{1,2,3}$ Zhong Yang $\mathbb{D}^{1,2,3}$ Zhao Zhang, ${ }^{1,2,3}$ Hao Xu $\mathbb{D},{ }^{1,2,3}$ Jiying Wu, ${ }^{1,2,3}$ \\ Dongsheng Zhou, ${ }^{1,2,3}$ Luwei Liao, ${ }^{1,2,3}$ and Qiuyan Zhang $\mathbb{\circledR}^{4}$ \\ ${ }^{1}$ College of Automation Engineering, Nanjing University of Aeronautics and Astronautics, Nanjing 211106, China \\ ${ }^{2}$ Key Laboratory of Navigation, Control and Health-Management Technologies of Advanced Aerocraft \\ (Nanjing University of Aeronautics and Astronautics), Ministry of Industry and Information Technology, \\ Nanjing 211106, China \\ ${ }^{3}$ Jiangsu Key Laboratory of Internet of Things and Control Technologies (Nanjing University of Aeronautics and Astronautics), \\ Nanjing 211106, China \\ ${ }^{4}$ Electric Power Research Institute of Guizhou Power Grid Co., Ltd., Guiyang 550002, China
}

Correspondence should be addressed to Zhong Yang; yangzhong@nuaa.edu.cn

Received 31 October 2020; Revised 29 November 2020; Accepted 16 December 2020; Published 28 December 2020

Academic Editor: M. Irfan Uddin

Copyright ( $\odot 2020$ Changliang Xu et al. This is an open access article distributed under the Creative Commons Attribution License, which permits unrestricted use, distribution, and reproduction in any medium, provided the original work is properly cited.

\begin{abstract}
Tree branches near the electric power transmission lines are of great threat to the electricity supply. Nowadays, the tasks of clearing threatening tree branches are still mostly operated by hand and simple tools. Traditional structures of the multirotor aerial robot have the problem of fixed structure and limited performance, which affects the stability and efficiency of pruning operation. In this article, in order to obtain better environmental adaptability, an active deformable trees-pruning aerial robot is presented. The deformation of the aerial robot is implemented through two ways, arm telescopic and folding. In order to suppress the influence of internal and external disturbances on the system, Active Disturbance Rejection Control (ADRC) technology is adopted to build the flight controller. Firstly, active deformation aerial robot structure is given, followed by system dynamic model establishment under wind disturbance using the Newton-Euler method. Also, the analysis of the gusts influence on the system is considered. Then, the active deformation aerial robot system is decoupled into a combination of six SISO systems, so that a disturbance rejection controller is designed. Finally, the expanded state observer and the nonlinear state error feedback law are used to inspect and compensate the disturbance. Simulation results of attitude and position tracking as well as the antidisturbance capability show that the active deformation aerial robot with the ADRC flight controller designed in this paper has excellent attitude control capabilities during flight and trees-pruning operation.
\end{abstract}

\section{Introduction}

With the feature of complexity, intelligent systems have significant research values and huge application potential $[1,2]$. In the giant and complex power grid, it has been always a tough issue that trees near the high-voltage transmission line channel often exceed the safe distance, causing the line to discharge through the trees to the ground, causing safety accidents such as power outages and fires, bringing huge inconvenience to social production and life. Therefore, the power sector consumes a lot of manpower and material resources every year for tree barrier cleaning. At present, most of its main methods use manpower, supplemented by simple tools such as hand saws. Not only is this kind of cleaning method limited in efficiency, but also human safety cannot be guaranteed. Casualties caused by such tasks in our country occur from time to time. Therefore, it is particularly necessary to develop and promote a set of efficient and safe tree barrier cleaning aerial robots that can replace manual cleaning methods.

The multirotor aerial robot that can take off and land vertically and hover in the air has the advantages of simple structure and good stability. The application research of using it as a work platform has attracted widespread attention [3-5].

However, the traditional structure of the multirotor aerial robot has the problem of fixed structure and limited performance, which affects the stability and efficiency of pruning operation. 
In response to the above-mentioned problems of conventional multirotor aircraft, researchers have studied improving the controllability of the aircraft by optimizing the relative direction of the rotors or using tiltable rotors. Paper [6] puts forward a new type of rotor installation plan in which eight rotors are arranged in a cube structure in different directions through the analysis of the force and moment of ordinary motors. Paper [7] combines the advantages of conventional multirotor systems and omnidirectional control aircraft and proposes a new type of flying platform. Papers [8,9] proposed a design scheme for a tiltable quadrotor and verified the effectiveness of the tiltable multirotor aircraft through simulation and actual flight experiments. Although these methods help achieve complex trajectories and operational tasks, they do not significantly change the shape of the multirotor aircraft frame.

On the contrary, only by directly changing the shape and structure of the multirotor aircraft during flight can this problem be effectively solved. Paper [10] focused on kinematics design and studied a new type of deformable quadrotor based on a scissor-like foldable structure, which can dynamically adjust the volume of the quadrotor to adapt to obstacles of various sizes and narrower space. Paper [11] proposed a deformable aircraft with four rotors connected in series, established its dynamic model, and designed an attitude LQI controller and a position PID controller to achieve the purpose of encircling the object and grasping it without additional gripping device. Paper [12] improved the diversity of aircraft deformation, divided the standard quadrotor structure into multiconnected platforms, and proposed a new control method that decoupled thrust control and rotor gimbal control and realized 3D folding. Paper [13] optimized the structure of the quadrotor and designed the automatic telescopic structure in the air and the manual folding structure after landing. The rational selection of materials and the strength check ensure the rationality of the airframe and can achieve the expected functions. Paper [14] designed a miniature deformable quadrotor, which achieved the purpose of deformation by using flexible materials to manufacture aircraft brackets and designing torsion spring and gear deformation mechanism. PID was selected as its flight control method.

The active deformation quadrotor is an aircraft that can actively change its shape during flight. Its shape changes have a strong influence on the mechanical properties, especially the expansion and folding of the arms, which not only directly change the position of the center of gravity and the inertia tensor of the aircraft but also change the mapping relationship between the lift generated by a single motor and the force and moment of the aircraft body [15]. This makes the active deformable quadrotor more uncertain and more coupled than the conventional quadrotor. In addition, the disturbance of the external environment during flight is inevitable, which makes the design of its controller more difficult. Researcher Han Jingqing inherited the essence of classic PID controllers and proposed Active Disturbance Rejection Control (ADRC) technology that requires low model accuracy and is easy to implement. ADRC has the advantages of fast control response speed and no overshoot, and transition process can be arranged. Also, the parameter adaptation range is large and has the ability to observe and compensate for disturbances inside and outside the system [16-18]. Because ADRC has obvious advantages in solving the control problems of nonlinear models with uncertainties and strong disturbances [19], researchers have tried ADRC on conventional quadrotor and verified the antidisturbance capability and robustness of the controller [20, 21]. Active Disturbance Rejection Control (ADRC) technology is a new type of control theory that does not require accurate modelling of the controlled object. A major feature of this theory is that it could effectively suppress the internal and external disturbances of the system, because it is able to estimate and compensate for the internal disturbances of the system and the unknown disturbances of the external environment in real time. During the operation of the tree barrier cleaning robot, the external force caused by the contact operation is a huge disturbance to the body, which brings about great challenges to the attitude stability control. In addition, ADRC has the characteristic of small amount of calculation, which makes it easy to realize realtime control of the robot's pose on the MCU (Microcontroller Unit).

With the purpose of pruning trees near the power transmission line, this paper proposes an active deformable aerial robot to improve environmental adaptability. The deformation forms are divided into arm telescopic and folding. In order to better suppress the internal and external disturbances of the system, this paper designs a flight control method based on Active Disturbance Rejection Control technology. First, the structural design is carried out, followed by the establishment of the dynamic model. Then analysis and calculation of the changes in the center of gravity and inertia matrix caused by the deformation are presented. Second, the system is decoupled and the attitude ADRC controller is designed. Then, the control distribution matrix form is given. Finally, through simulation, the deformation ability of the designed active deformation aerial robot and the effectiveness, anti-interference performance, and robustness of the ADRC controller are verified.

\section{Structural Design of Active Deformation Aerial Robot}

The active deformation aerial robot designed in this paper is mainly composed of the following four parts: (1) the centered fuselage, (2) the deformable arm, (3) the motor and the rotor, and (4) deformation of the arm to achieve the aerial robot deforming various steering gears. Assume that the deformable aerial robot is a rigid body, as shown in Figure 1. The earth fixed coordinate system $O_{E} x_{E} y_{E} z_{E}$ is defined to be fixed to the ground, and the origin of the body coordinate system $O_{B} x_{B} y_{B} z_{B}$ is fixed at the center of the robot fuselage. At the same time, the telescopic rudder of the active deformable active aerial robot is defined. The engine is installed at the midpoint of the arm, and the rotary servo is installed on the fuselage. For the convenience of writing, this article 


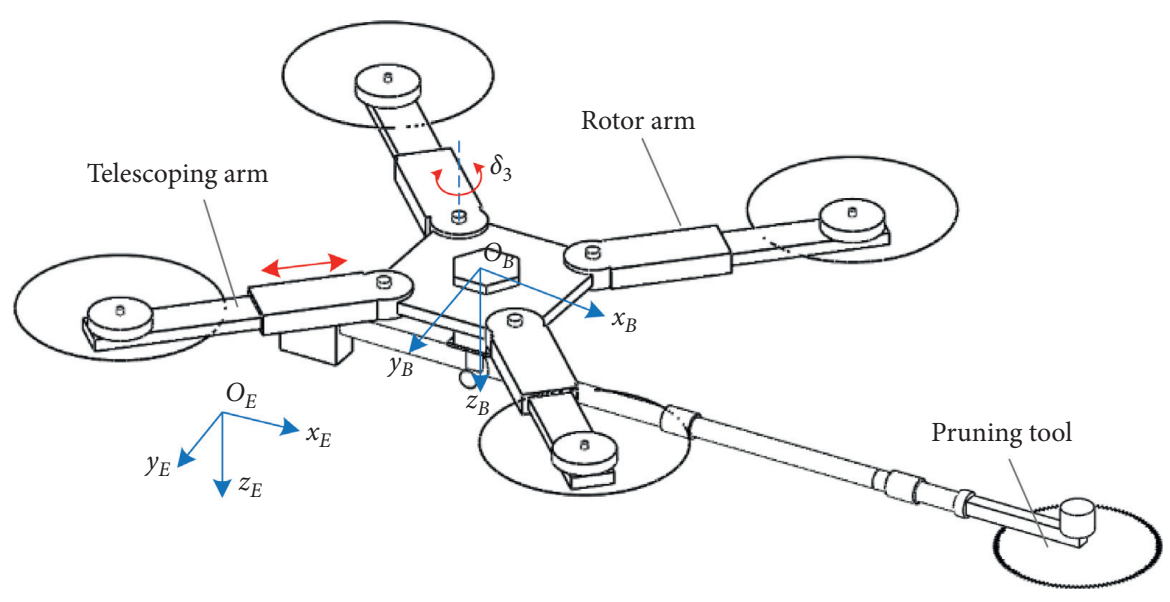

FIgURE 1: Frames of actively deformable aerial robot.

uses $i$ th to represent the $i$ th, $i \in\{1,2,3,4\}$. Define $l_{i} \in[15 \mathrm{~cm}, 25 \mathrm{~cm}]$ as the length of $i$ th retractable arm. Define $\delta_{i}$ as the rotation angle of the $i$ th arm around the $z_{B}$ axis of the machine system. When the $i$ th arm rotates clockwise, $\delta_{i}$ is positive. Only $\delta_{3}$ is shown in Figure 1. The numbers of the arms and motors have been marked in the figure. Motors nos. 1 and 3 rotate counterclockwise, and motors nos. 2 and 4 rotate clockwise.

The active deformation aerial robot has two deformation functions. (1) The arm is stretched and deformed by the telescopic steering gear; that is, the length of the arm is changed. (2) The arm is folded and deformed around the $z_{B}$ axis of the machine system by rotating the steering gear changing $\delta_{i}$. Its four arms can be deformed individually or in combination. In the following of the paper, expansion and folding are used to represent these two kinds of deformation.

2.1. Kinematics and Dynamics. This article uses ${ }^{(\cdot)} R_{(\cdot)} \in \mathrm{SO}(3)$ to represent the rotation matrix between coordinate systems, and ${ }^{E} R_{B}$ represents the rotation matrix from the machine system to the earth's fixed coordinate system (in the text, $s_{(\cdot)}$ means $\sin _{(\cdot)}, c_{(\cdot)}$ means $\cos (\cdot)$, and $t_{(\cdot)}$ means $\left.\tan _{(\cdot)}\right)$ :

$$
{ }^{E} \mathbf{R}_{B}=\left[\begin{array}{ccc}
c_{\theta} c_{\psi} & s_{\phi} s_{\theta} c_{\psi}-c_{\phi} s_{\psi} & c_{\phi} s_{\theta} c_{\psi}+s_{\phi} s_{\psi} \\
c_{\theta} s_{\psi} & s_{\phi} s_{\theta} s_{\psi}+c_{\phi} c_{\psi} & c_{\phi} s_{\theta} s_{\psi}-s_{\phi} c_{\psi} \\
-s_{\theta} & s_{\phi} c_{\theta} & c_{\phi} c_{\theta}
\end{array}\right] .
$$

The rigid body kinematics model of the actively deformed aerial robot is

$$
\left\{\begin{array}{l}
\dot{P}^{E}=V^{E}, \\
\dot{\Theta}=W \Omega^{B},
\end{array}\right.
$$

where $P^{E}=[x ; y ; z]$ and $V^{E}=[u ; v ; w]$, respectively, represent the airframe position and triaxial velocity of the robot in the earth's fixed coordinate system, $\Theta=[\phi ; \theta ; \psi]$ represents the Euler angle of the robot, and $\Omega^{B}=[p ; q ; r]$ represents the triaxial angular velocity of the robot in the airframe coordinate system.

$$
\mathbf{W}=\left[\begin{array}{ccc}
1 & t_{\theta} s_{\phi} & t_{\theta} c_{\phi} \\
0 & c_{\phi} & -s_{\phi} \\
0 & \frac{s_{\phi}}{c_{\theta}} & \frac{c_{\phi}}{c_{\theta}}
\end{array}\right] .
$$

The force and moment changes caused by the deformation of the active deforming aerial robot are more complicated than the conventional aerial robots. The dynamic model of the active deforming aerial robot is analyzed and established below.

The fuselage is regarded as a mass point during the deformation process. As a six-degree-of-freedom rigid body, the dynamic model of the actively deformed aerial robot is obtained by Newton-Euler equations [22]. In this paper, the system translation equation is defined in the earth's fixed coordinate system, and the rotation equation is defined in the body coordinate system:

$$
\left[\begin{array}{c}
m \dot{V}^{E} \\
I_{B} \dot{\Omega}^{B}
\end{array}\right]+\left[\begin{array}{c}
0 \\
\Omega^{B} \times I_{B} \Omega^{B}
\end{array}\right]=\left[\begin{array}{c}
F^{E} \\
M^{B}
\end{array}\right],
$$

where $m$ represents the mass of the machine body and $I_{B}$ is the inertial matrix. Its value will change due to the deformation of the body, which will be analyzed in detail later.

The force analysis of the active deformation aerial robot system is carried out, and the following is obtained:

$$
\left\{\begin{array}{l}
F^{E}=G^{E}+F_{t}^{E}+F_{\text {wind }}^{E}+D^{E}, \\
F_{t}^{E}={ }^{E} R_{B} \sum_{i=1}^{4} T_{i}, \\
\mathbf{T}_{i}=\left[\begin{array}{lll}
0 & 0 & -k_{f} n_{i}^{2}
\end{array}\right]^{T} .
\end{array}\right.
$$

where $G^{E}$ represents gravity. $F_{t}^{E}$ represents the lift generated by the rotor. $F_{\text {wind }}^{E}$ represents the wind disturbance. $D^{E}$ represents the air resistance. $k_{f}$ is the lift coefficient of the rotor, and $n_{i}$ is the speed of the $i$ th rotor.

Before analyzing the torque experienced by the active deformable aerial robot, the position of the center of mass 
and the inertia matrix are first analyzed. Compared with conventional quadrotors, the position of the center of mass and the inertia matrix of the actively deformed aerial robots will change due to the expansion and folding of the arms. Therefore, when the system is deformed, the position of the center of mass and the inertia matrix of the system need to be recalculated. The offset between the center of mass of the active deformed aerial robot and the origin of the system coordinates [23] $r_{\mathrm{CoG}} \in R^{3}$ is expressed as follows:

$$
\left\{\begin{array}{l}
r_{\mathrm{CoG}}=\frac{m_{\mathrm{body}} r_{\mathrm{body}}+A}{m_{\mathrm{body}}+\sum_{i=1}^{4}\left(m_{\mathrm{arm}}+m_{\mathrm{mot}}+m_{\mathrm{rot}}+m_{\mathrm{elo}}\right)}, \\
A=\sum_{i=1}^{4}\left(m_{\mathrm{arm}} r_{\mathrm{arm}, i}+m_{\mathrm{mot}} r_{\mathrm{mot}, i}+m_{\mathrm{rot}} r_{\mathrm{rot}, i}+m_{\mathrm{elo}} r_{\mathrm{elo}, i}\right)
\end{array}\right.
$$

where $m_{(\cdot)}$ represents the mass of $(\cdot)$ and $r_{(\cdot)}$ represents the vector diameter from the origin of the machine system coordinates to (.). The meaning of each subscript English letter is shown in Table 1.

For the body inertia matrix $I_{B}$ of an actively deformed aerial robot, the parallel axis theorem can be used for calculation. Here are some assumptions: the fuselage containing the rotating steering gear is regarded as a rectangular parallelepiped with length and width of $l_{\text {body }}$ and height of $h_{\text {body }}$. The cuboid length, width, and height are $l_{\text {arm }, i}, w_{\text {arm }}$, and $h_{\mathrm{arm}}$, and the rotor and the motor are regarded as cylinders, in which the rotor radius is $r_{\text {mot }}$, the height is $h_{\text {mot }}$, the motor radius is $r_{\text {rot }}$, and the height is $h_{\text {rot }}$; the telescopic steering gear is regarded as the cuboid with length, width, and height, respectively, of $l_{\mathrm{elo}}, w_{\mathrm{elo}}$, and $h_{\mathrm{elo}}$. The calculation formula for the moment of inertia of each part [24] is as follows:

$$
\left\{\begin{array}{l}
I_{\text {body }}=\frac{m_{\text {body }}}{12} \operatorname{diag}\left(h_{\text {body }}^{2}+l_{\text {body }}^{2}, h_{\text {body }}^{2}+l_{\text {body }}^{2}, l_{\text {body }}^{2}+l_{\text {body }}^{2}\right), \\
\widetilde{I}_{\mathrm{arm}, i}=\frac{m_{\mathrm{arm}}}{12} \operatorname{diag}\left(w_{\mathrm{arm}}^{2}+h_{\mathrm{arm}}^{2}, h_{\mathrm{arm}}^{2}+l_{\mathrm{arm}, i}^{2}, w_{\mathrm{arm}}^{2}+l_{\mathrm{arm}, i}^{2}\right), \\
I_{\mathrm{mot}}=\frac{m_{\mathrm{mot}}}{12} \operatorname{diag}\left(3 r_{\mathrm{mot}}^{2}+h_{\mathrm{mot}}^{2}, 3 r_{\mathrm{mot}}^{2}+h_{\mathrm{mot}}^{2}, 6 r_{\mathrm{mot}}^{2}\right), \\
I_{\mathrm{rot}}=\frac{m_{\mathrm{rot}}}{12} \operatorname{diag}\left(3 r_{\mathrm{rot}}^{2}+h_{\mathrm{rot}}^{2}, 3 r_{\mathrm{rot}}^{2}+h_{\mathrm{rot}}^{2}, 6 r_{\mathrm{rot}}^{2}\right), \\
\widetilde{I}_{\mathrm{elo}, i}=\frac{m_{\text {elo }}}{12} \operatorname{diag}\left(w_{\mathrm{elo}}^{2}+h_{\mathrm{elo}}^{2}, h_{\mathrm{elo}}^{2}+l_{\mathrm{elo}}^{2}, w_{\mathrm{elo}}^{2}+l_{\mathrm{elo}}^{2}\right),
\end{array}\right.
$$

where $I_{(\cdot)}$ represents the inertia value of (.).

Considering that the motor, rotor, arm, and telescopic steering gear are actively deforming the aerial robot during folding and deformation, inertia must also be recalculated [25]. Since the inertia of the cylinder does not change when it is folded and deformed, this change can be ignored for the inertia of the motor and the rotor. Since the fuselage structure is fixedly
Table 1: Meaning of subscript letter.

\begin{tabular}{lc}
\hline Subscripts & Descriptions \\
\hline body & Robot body \\
arm,$i$ & $i$ th robot propeller arm \\
mot, $i$ & $i$ th motor \\
rot, $i$ & $i$ th rotation \\
elo, $i$ & $i$ th telescopic steering gear \\
\hline
\end{tabular}

connected to the fuselage coordinate system, the inertia of the fuselage does not need to be changed. Therefore, the rotation matrix is introduced to rerepresent them:

$$
\left\{\begin{array}{l}
I_{\mathrm{arm}, i}=R_{z}\left(\delta_{i}\right) \widetilde{I}_{\mathrm{arm}, \mathrm{i}} R_{z}\left(\delta_{i}\right)^{T} \\
I_{\mathrm{elo}, i}=R_{z}\left(\delta_{i}\right) \widetilde{I}_{\mathrm{elo}, i} R_{z}\left(\delta_{i}\right)^{T}
\end{array}, \quad i \in\{1,2,3,4\} .\right.
$$

The rotation matrix is expressed as follows:

$$
\mathbf{R}_{z}\left(\delta_{i}\right)=\left[\begin{array}{ccc}
c_{\delta_{i}} & s_{\delta_{i}} & 0 \\
-s_{\delta_{i}} & c_{\delta_{i}} & 0 \\
0 & 0 & 1
\end{array}\right] \text {. }
$$

So, the inertia calculation formula can be expressed as

$$
\begin{aligned}
I_{B}= & I_{\mathrm{body}}-m_{\mathrm{body}}\left(r_{\mathrm{body}}-r_{\mathrm{CoG}}\right)^{2} \\
& +\sum_{i=1}^{4}\left(I_{\mathrm{arm}, i}-m_{\mathrm{arm}}\left(r_{\mathrm{arm}, i}-r_{\mathrm{CoG}}\right)^{2}\right. \\
& +I_{\mathrm{mot}}-m_{\mathrm{mot}}\left(r_{\mathrm{mot}, i}-r_{\mathrm{CoG}}\right)^{2} \\
& \left.+I_{\mathrm{rot}}-m_{\mathrm{rot}}\left(r_{\mathrm{rot}, i}-r_{\mathrm{CoG}}\right)^{2}+I_{\mathrm{elo}, i}-m_{\mathrm{elo}}\left(r_{\mathrm{elo}, i}-r_{\mathrm{CoG}}\right)^{2}\right) .
\end{aligned}
$$

Analyzing the moment of the active deforming aerial robot, we get

$$
M^{B}=M_{t}^{B}+M_{\mathrm{anti}}^{B}+M_{\mathrm{gyro}}^{B}+M_{\mathrm{wind}}^{B}+M_{G}^{B},
$$

where $M_{t}^{B}$ is the torque generated by the lift of the rotor, $M_{\text {anti }}^{B}$ is the rotation reaction torque of the rotor, $M_{\text {gyro }}^{B}$ is the system gyro effect item, and $M_{\text {wind }}^{B}$ is the wind disturbance moment. Different from the conventional quadrotor, there is an additional gravitational moment $M_{G}^{B}$.

The torque generated by the rotor thrust is

$$
\mathbf{M}_{t}^{B}=\sum_{i=1}^{4}\left(L_{i} \times T_{i}\right),
$$

where $L_{i}$ represents the coordinate vector of the lift application point of the ithrotor in the robot system. The expression is as follows:

$$
L_{i=}\left\{\begin{array}{lll}
l_{i}\left[\begin{array}{lll}
-c_{\alpha_{i}+(i-1 / 2) \pi} & s_{\alpha_{i}+(i-1 / 2) \pi} & 0
\end{array}\right]^{T}, & i \in\{1,3\}, \\
l_{i}\left[\begin{array}{lll}
-s_{\alpha_{i}+(i-2 / 2) \pi} & -c_{\alpha_{i}+(i-2 / 2) \pi} & 0
\end{array}\right]^{T}, & i \in\{2,4\},
\end{array}\right.
$$

where $\alpha_{i}=(\pi / 4)-\delta_{i}$ is converted to angle system, namely, $\alpha_{i} \in\left[0^{\circ}, 90^{\circ}\right]$. 
The antitorque expression is

$$
\left\{\begin{array}{l}
\mathbf{M}_{\text {anti }}^{B}=\left[\begin{array}{lll}
0 & 0 & \sum_{i=1}^{4} Q_{i}
\end{array}\right]^{T}, \\
Q_{i}=\left\{\begin{array}{ll}
k_{m} n_{i}^{2} & i \in\{1,3\} \\
-k_{m} n_{i}^{2} & i \in\{2,4\}
\end{array}, \quad k_{m}>0 .\right.
\end{array}\right.
$$

The system gyro effect is mainly caused by the rotation of the body:

$$
\left\{\mathbf{M}_{\text {gyro }}^{B}=J_{P} \sum_{i=1}^{4}\left(\Omega^{B} \times(-1)^{f(i)}\left[\begin{array}{l}
0 \\
0 \\
n_{i}
\end{array}\right]\right), f(i)= \begin{cases}1, & i \in\{1,3\}, \\
0, & i \in\{2,4\} .\end{cases}\right.
$$

The expression of gravitational moment is

$$
M_{G}^{B}=r_{\mathrm{CoG}} \times\left({ }^{B} R_{E} G^{E}\right) .
$$

2.2. Wind Disturbance Modelling. The influence of the wind field environment on the multirotor aircraft is partly caused by the aerodynamic effect of the rotor and is also partly caused by the air resistance of the frame of the multirotor aircraft [26]. The active deformation aerial robot will inevitably be affected by the external wind field when it is flying, especially when it is deformed. So, wind gusts will have a certain impact on it. The wind disturbance model can calculate the forces and moments acting on the actively deformed aerial robot in a given wind field, so modelling and analysis of wind disturbance are essential [27].

The schematic diagram of the aerodynamic effect of the rotor under the action of the wind field is shown in Figure 2. In the figure, $V_{\text {wind }}$ represents the wind speed, $V_{d}$ represents the induced speed, and $V_{\text {total }}$ represents the vector sum of the wind speed and the induced speed.

According to the rotor slip flow theory [28], the calculation formula of induced speed is as follows:

$$
\left\|V_{t}\right\|=\sqrt{\frac{k_{f} n^{2}}{2 \pi \rho r^{2}}}
$$

where $\rho$ represents the local air density and $r$ represents the radius of the rotor blade. When there is a wind field, the total aerodynamic force experienced by a single rotor [29] is

$$
\left\|T_{i}+F_{w_{i}}\right\|=2 \pi \rho r^{2}\left\|V_{t}\right\|\left\|V_{\text {wind }}+V_{t}\right\|, \quad i \in\{1,2,3,4\} .
$$

So, the wind disturbance force and wind disturbance moment of the $i$ th rotor are as follows:

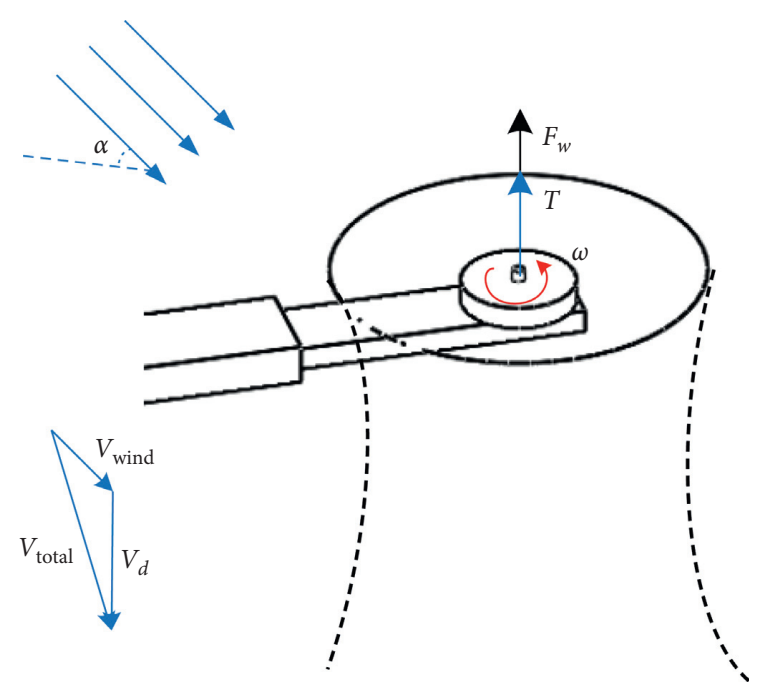

FIgURE 2: Aerodynamics of rotor under wind gust.

$$
\begin{aligned}
& F_{w_{i}}=k_{f} n_{i}^{2}-2 \pi \rho r^{2}\left\|\left[\begin{array}{c}
0 \\
0 \\
\sqrt{\frac{k_{f} n^{2}}{2 \pi \rho r^{2}}}
\end{array}\right]+{ }^{B} R_{E} V_{\text {wind }}^{E}\right\| \text {, } \\
& M_{w_{i}}= \begin{cases}-\frac{k_{m}}{k_{f}} F_{w_{i}}, & i \in\{1,3\}, \\
\frac{k_{m}}{k_{f}} F_{w_{i}}, & i \in\{2,4\} .\end{cases}
\end{aligned}
$$

Thereby,

$$
\mathbf{F}_{\text {wind }}^{E}={ }^{E} \mathbf{R}_{B} \sum_{i=4}^{4}\left[\begin{array}{c}
0 \\
0 \\
F_{w_{i}}
\end{array}\right] \text {, }
$$

$$
\mathbf{M}_{\text {wind }}^{B}=\sum_{i=4}^{4}\left[\begin{array}{c}
0 \\
0 \\
M_{w_{i}}
\end{array}\right] .
$$

The air resistance calculation formula is

$$
D^{E}=\frac{1}{2} c \rho S_{\text {air }} V_{\text {air }}^{2},
$$

where $c$ represents the coefficient of air resistance, $S_{\text {air }}$ represents the windward area, and $V_{\text {air }}$ represents the relative speed of the robot and the air; that is, $V_{\text {air }}=V_{w}^{E}-V^{E}$. When calculating the windward area of a deformable robot, the robot is simplified as a cylinder, and the average windward area is taken as $\bar{S}=\sigma 2 \pi \bar{l} h+(1-\sigma) \pi \bar{l}^{2}$, where $\sigma \in(0,1)$ is the crosswind coefficient, $h$ is the height of the robot body, and $\bar{l}$ is the average length of the arms, so 


$$
D^{E}=\frac{1}{2} c \rho \bar{S}_{\text {air }} V_{\text {air }}\left\|V_{\text {air }}\right\|
$$

2.3. Control Distribution. Like the conventional quadrotor, the active deformation aerial robot system control force and moment are mainly the thrust and reaction torque generated by the rotor. Combining equations (4)-(24), $U_{F}^{E}$ and $U_{M}^{B}$ are used to represent the force and torque generated by the rotor. The dynamic model can be reexpressed:

$$
\left[\begin{array}{c}
m \dot{V}^{E} \\
I_{B} \dot{\Omega}^{B}+\Omega^{B} \times I_{B} \Omega^{B}
\end{array}\right]=\left[\begin{array}{c}
G^{E}+F_{\text {wind }}^{E}+D^{E}+U_{F}^{E} \\
M_{\text {gyro }}^{B}+M_{\text {wind }}^{B}+M_{G}^{B}+U_{M}^{B}
\end{array}\right] .
$$

The relationship between $U_{F}^{E}$ and $U_{M}^{B}$ and rotor speed $n$ is

$$
\begin{aligned}
{\left[\begin{array}{c}
U_{F}^{E} \\
U_{M}^{B}
\end{array}\right]=C\left[\begin{array}{l}
n_{1}^{2} \\
n_{2}^{2} \\
n_{3}^{2} \\
n_{4}^{2}
\end{array}\right], } \\
\mathbf{C}=\left[\begin{array}{cccc}
k_{f} & k_{f} & k_{f} & k_{f} \\
-k_{f} l_{1} c_{\alpha_{1}} & -k_{f} l_{2} s_{\alpha_{2}} & k_{f} l_{3} c_{\alpha_{3}} & k_{f} l_{4} s_{\alpha_{4}} \\
k_{f} l_{1} s_{\alpha_{1}} & -k_{f} l_{2} c_{\alpha_{2}} & -k_{f} l_{3} s_{\alpha_{3}} & k_{f} l_{4} c_{\alpha_{4}} \\
k_{m} & -k_{m} & k_{m} & -k_{m}
\end{array}\right] .
\end{aligned}
$$

Matrix $C$ is the control efficiency matrix. It can be seen that matrix $C$ is a time-varying matrix. The following verification matrix $C$ is singular only at the singular point $\left(\alpha_{1}, \alpha_{2}, \alpha_{3}, \alpha_{4}\right) \in\left\{\left(0^{\circ}, 90^{\circ}, 0^{\circ}, 90^{\circ}\right),\left(90^{\circ}, 0^{\circ}, 90^{\circ}, 0^{\circ}\right)\right\}$, and the other matrices $C$ are all nonsingular.

Proof. Using the method of proof by contradiction and assuming that the square matrix $C$ is a singular matrix, its columns must not be full of rank. Because of $k_{f}, k_{m} \neq 0$, only column 1 and column 3 or column 2 and column 4 are proportional. Let $C_{i j}$ be the element in row $i$ and column $j$ for discussion:

(1) Assuming that the sine and cosines of all angles are not 0 , column 1 and column 3 are proportional. $C_{11} / C_{13}=C_{41} / C_{43}=1$, so $-k_{f} l_{1} c_{\alpha_{1}} / k_{f} l_{3} c_{\alpha_{3}}=1$, that is, $\quad c_{\alpha_{1}} / c_{\alpha_{3}}=-l_{3} / l_{1}$, and $s_{\alpha_{1}} / s_{\alpha_{3}}=-l_{3} / l_{1}$ derives $c_{\alpha_{1}} / c_{\alpha_{3}}=s_{\alpha_{1}} / s_{\alpha_{3}}$, which is further written as $c_{\alpha_{1}} s_{\alpha_{3}}-$ $s_{\alpha_{1}} c_{\alpha_{3}}=0$, that is, $s_{\alpha_{1}-\alpha_{3}}=0$, which is also equivalent to $\alpha_{1}-\alpha_{3}=k \pi, k \in Z$. At this time, only the situation where $\alpha_{1}=\alpha_{3}=0^{\circ}$ exists, but it is contrary to the assumption, so there is no solution.

(2) Assuming that the sine and cosines of all angles are not 0 , column 2 and column 4 are proportional: with the same principle, there is no solution.

(3) Suppose that there are an angular sine and cosine of 0 : when $c_{\alpha}=0$ or $s_{\alpha}=0$, $\alpha$ is $0^{\circ}$ or $90^{\circ}$, and the empirical calculation is that only in the case of $\left(\alpha_{1}, \alpha_{2}, \alpha_{3}, \alpha_{4}\right) \in\left\{\left(0^{\circ}, 90^{\circ}, 0^{\circ}, 90^{\circ}\right),\left(90^{\circ}, 0^{\circ}, 90^{\circ}, 0^{\circ}\right)\right\}$ is matrix $C$ not full of rank, which is a singular matrix.

Proof ends.

Since the deformation process of the actively deformed aerial robot in this paper does not involve the location of singular points, it can be considered that matrix $C$ is fully reversible. So, the form of control distribution is as follows:

$$
\left[\begin{array}{l}
n_{1}^{2} \\
n_{2}^{2} \\
n_{3}^{2} \\
n_{4}^{2}
\end{array}\right]=C^{-1}\left[\begin{array}{c}
U_{F}^{E} \\
U_{M}^{B}
\end{array}\right] .
$$

It can be seen from equations (25)-(28) that the active deformation aerial robot model has strong nonlinearity and coupling. The deformation causes the length and angle of the arm to change, which leads to the change of model parameters. Compared with the conventional aerial robot, the internal disturbance is greater. At the same time, external disturbances such as the wind field experienced by the actively deformed aerial robot cannot be ignored.

\section{Design of Active Disturbance Rejection Flight Control Law}

One of the significant features of Active Disturbance Rejection Control technology is that it has low dependence on the accuracy of the system modelling. It could estimate and dynamically compensate for the internal and external disturbances of the system as well, which contributes to the system's disturbance rejection and robustness ability. In this paper, Active Disturbance Rejection Control algorithms are implemented using the following three parts: tracking differentiator (TD), extended state observer (ESO), and nonlinear state error feedback (NLSEF).

3.1. Preliminary of the ARDCLaw Design. There is a coupling between the position and attitude of the actively deformed aerial robot studied in this paper, which is an underactuated system. Only six-degree-of-freedom motion can be achieved in the incomplete sense, and the roll and pitch angles need to be changed while doing horizontal motion [30]. ADRC provides a more convenient way for the decoupling control of the multivariable coupling system, in which 6 states in the dynamic model of the active deformation aerial robot system are regarded as 6 channels, and ADRC can cause the coupling and deformation between the channels of the system. The parameter perturbation is treated as internal disturbance, and ESO is used to estimate and compensate for the internal and external disturbances of the system to realize the decoupling of the state of each channel. The virtual control quantity is introduced to realize the decoupling of system control, thereby converting the system description form from MIMO to six SISO systems combination. Therefore, the system's six-degree-of-freedom dynamic model equation (25) is rewritten in the following form: 


$$
\left\{\begin{array}{l}
\ddot{x}=s_{1}\left(x, \dot{x}, \omega_{\text {out }_{1}}, \omega_{\mathrm{def}_{1}}\right)+b_{1} u_{1}, \\
\ddot{y}=s_{2}\left(y, \dot{y}, \omega_{\text {out }_{2}}, \omega_{\text {def }_{2}}\right)+b_{2} u_{2}, \\
\ddot{z}=s_{3}\left(z, \dot{z}, \omega_{\text {out }_{3}}, \omega_{\text {def }_{3}}\right)+b_{3} u_{3}, \\
\ddot{\phi}=s_{4}\left(p, q, r, \phi, \dot{\phi}, \theta, \dot{\theta}, \psi, \dot{\psi}, \omega_{\text {out }_{4}}, \omega_{\text {def }_{4}}\right)+b_{4} u_{4}, \\
\ddot{\theta}=s_{5}\left(p, q, r, \phi, \dot{\phi}, \theta, \dot{\theta}, \psi, \dot{\psi}, \omega_{\text {out }_{5}}, \omega_{\text {def }_{5}}\right)+b_{5} u_{5}, \\
\ddot{\psi}=s_{6}\left(p, q, r, \phi, \dot{\phi}, \theta, \dot{\theta}, \psi, \dot{\psi}, \omega_{\text {out }_{6}}, \omega_{\text {def }_{6}}\right)+b_{6} u_{6},
\end{array}\right.
$$

where $s_{i}(\cdot)$ is the uncertainty item and $\omega_{\text {out }}$ and $\omega_{\text {def }_{i}}$ are the disturbances caused by the external disturbance and deformation of the system, respectively. $\left(b_{1}, b_{2}, b_{3}\right)$ is an adjustable parameter with a size near $(1 / m) .\left(b_{4}, b_{5}, b_{6}\right)$ is an adjustable parameter with a size near $\left(\left(1 / I_{x x}\right),\left(1 / I_{y y}\right),\left(1 / I_{z z}\right)\right)$, and $\left(u_{1}, u_{2}, u_{3}, u_{4}, u_{5}, u_{6}\right)$ is the introduced virtual control quantity.

Based on this, the control law adopts an inner and outer loop strategy, where the inner loop is attitude control and the outer loop is position control. The introduction of the control quantity $\left(U_{1}, U_{2}, U_{3}, U_{4}\right)$, respectively, represents the expected value of the total lift and the expected value of the torque around the three axes of the machine system. The actual position value $(x, y, z)$ and the position expected value $\left(x^{d}, y^{d}, z^{d}\right)$ are used as the input of the outer loop position ADRC control law, and the expected values of roll and pitch angles $\left(\phi^{d}, \theta^{d}\right)$ and $U_{1}$ are output. The actual attitude value $(\phi, \theta, \psi)$ and the expected attitude value $\left(\phi^{d}, \theta^{d}, \psi^{d}\right)$ are input to the ADRC control law of the inner loop attitude. The output is $\left(U_{2}, U_{3}, U_{4}\right)$. Finally, $\left(U_{1}, U_{2}, U_{3}, U_{4}\right)$ obtains the desired motor speed $\left(n_{1}^{d}, n_{2}^{d}, n_{3}^{d}, n_{4}^{d}\right)$ through control distribution, where the conversion relations between the virtual control variables $\left(u_{1}, u_{2}, u_{3}, u_{4}, u_{5}, u_{6}\right)$ and $\left(U_{1}, \phi^{d}, \theta^{d}\right)$ are

$$
\begin{aligned}
& \left\{\begin{array}{l}
U_{1}=\sqrt{\left(m u_{1}\right)^{2}+\left(m u_{2}\right)^{2}+\left(m u_{3}-m g\right)^{2}}, \\
\phi^{d}=-\arcsin \frac{m u_{1} s_{\psi}-m u_{2} c_{\psi}}{U_{1}}, \\
\theta^{d}=-\arcsin \frac{m u_{1} c_{\psi}+m u_{2} s_{\psi}}{U_{1} c_{\phi^{d}}}, \\
{\left[\begin{array}{l}
U_{2} \\
U_{3} \\
U_{4}
\end{array}\right]=I_{B} W^{-1}\left[\begin{array}{l}
u_{4} \\
u_{5} \\
u_{6}
\end{array}\right] .}
\end{array}\right.
\end{aligned}
$$

\subsection{Design of Attitude Active Disturbance Rejection Control} Law. Take the pitch angle $\theta$ channel as an example to illustrate the design process of the attitude ADRC control law:
(1) Design a tracking differentiator (TD) to arrange the transition process with the given signal $\theta^{d}$ as a reference input:

$$
\left\{\begin{array}{l}
e=v_{1}-\theta_{d}, \\
\mathrm{fh}=\text { fhan }\left(e, v_{2}, r, h\right), \\
v_{1}=v_{1}+h v_{2}, \\
v_{2}=v_{2}+\mathrm{hfh},
\end{array}\right.
$$

where $v_{1}$ is the transition value of TD from the initial value to $\theta^{d} . v_{2}$ is the derivative value of $v_{1}$, and the parameters $(r, h)$ are the fast factor and the filter factor, respectively.

(2) Design an extended state observer (ESO) to use the system output $\theta$ and control input $u_{5}$ to observe the system state and disturbances in real time:

$$
\left\{\begin{array}{l}
e=z_{1}-\theta \\
\mathrm{fe}=\mathrm{fal}(e, 0.5, \delta) \\
\mathrm{fe}_{1}=\mathrm{fal}(e, 0.25, \delta) \\
z_{1}=z_{1}+h\left(z_{2}-\beta_{01} e\right) \\
z_{2}=z_{2}+h\left(z_{3}-\beta_{02} f e+b_{5} u_{5}\right) \\
z_{3}=z_{3}+h\left(-\beta_{03} \mathrm{fe}_{1}\right)
\end{array}\right.
$$

where $\left(z_{1}, z_{2}\right)$ tracks $(\theta, \dot{\theta}), z_{3}$ estimates the total disturbance $s_{5}$, and $\left(\beta_{01}, \beta_{02}, \beta_{03}\right)$ is a set of adjustable parameters.

(3) Design the nonlinear state error feedback (NLSEF) law, calculate $u_{0}$, and combine it with disturbance compensation to calculate the control variable $u_{5}$ :

$$
\left\{\begin{array}{l}
e_{1}=v_{1}-z_{1}, \\
e_{2}=v_{2}-z_{2}, \\
u_{0}=- \text { fhan }\left(e_{1}, c e_{2}, r, h\right), \\
u_{5}=u_{0}-\frac{z_{3}}{b_{5}}
\end{array}\right.
$$

The parameter $(r, h, c)$ is an adjustable parameter.

The expressions of the fastest tracking control integrated function fhan $\left(x_{1}, x_{2}, r, h\right)$ and nonlinear function fal $(e, \alpha, \delta)$ are as follows: 


$$
\begin{aligned}
& \quad \operatorname{fal}(e, \alpha, \delta)= \begin{cases}\operatorname{sign}(e)|e|^{\alpha}, & |e| \leq \delta, \\
\delta^{1-\alpha} e, & |e|>\delta,\end{cases} \\
& d_{0}=h d, \\
& y=x_{1}+h x_{2}, \\
& a_{0}=\sqrt{d^{2}+8 r|y|}, \\
& a= \begin{cases}x_{2}+0.5\left(a_{0}-d\right) \operatorname{sign}(y), & |y|>d_{0}, \\
x_{2}+\frac{y}{h}, & |y| \leq d_{0},\end{cases} \\
& \text { fhan }= \begin{cases}-r \operatorname{sign}(a), & |a|>d, \\
\frac{r a}{d}, & |a| \leq d,\end{cases}
\end{aligned}
$$

where $\delta>0$. The design of the $\phi$ and $\psi$ channel control law is similar to that of the $\theta$ channel and will not be described in detail here.

\subsection{Position Active Disturbance Rejection Control Law Design.} During the design of the position ADRC control law, the design of the position's three-channel TD, ESO, and NLSEF is similar to the design of the $\theta$ channel in the previous section. The only difference is that the $z$ channel NLSEF adopts the following form:

$$
\left\{\begin{array}{l}
e_{1}=v_{1}-z_{1}, \\
e_{2}=v_{2}-z_{2}, \\
u_{0}=k_{1} e_{1}+k_{2} e_{2}, \\
u_{3}=u_{0}-\frac{z_{3}}{b_{3}},
\end{array}\right.
$$

where $\left(k_{1}, k_{2}\right)$ is an adjustable parameter.

\section{Simulation Verification}

In order to verify the rationality of the designed active deformable aerial robot and the effectiveness of its control law, simulations are arranged for verification in this section. First, we set up attitude response and position tracking experiments in order to verify the dynamic performance of the ADRC control algorithm. Then, in order to verify the control performance of the deformation function, experiments were arranged for the extension and folding of a single arm and two arms under the control of hovering, respectively. The folding experiments of the four arms are also arranged. Finally, the deformable aerial robot needs to perform cleaning operations, so the anti-interference performance is also one of the key points to be verified in this article. Therefore, an attitude control experiment of the robot under the action of wind disturbance and treespruning contact force is arranged. Based on MATLAB/ Simulink platform, the deformable aerial robot simulation built with simulation step is set to $0.001 \mathrm{~s}$, and the system simulation parameters are shown in Table 2.

The parameters of the active disturbance rejection flight controller are shown in Table 3. In this paragraph, we have presented some method and experience for the tuning of ADRC controller to obtain a satisfying control performance. There are two adjustable parameters of TD, which are $r$ and $h$. There are tricks that the larger $h$ is, the better the filtering effect of TD will be, and the greater phase delay will be. The larger $r$ is, the faster TD can track the input signal. Among the adjustable parameters of ESO, $\left(\beta_{01}, \beta_{02}, \beta_{03}\right)$ affects the convergence speed of ESO, which is generally set according to the empirical formula. The tuning of $b$ refers to equation (29). The meaning of $(r, h)$ in NLSEF is the same as that in TD. $c$ is the damping factor, and the meaning is the same as the damping coefficient of the second-order system. Increasing $c$ would make the system overshoot decrease but the response speed becomes slower. $\left(k_{1}, k_{2}\right)$ functions, respectively, equivalent to the proportional module and the differential module in classical PID control. Increasing $k_{1}$ increases the system's response speed but is prone to overshoot or even oscillation, while increasing $k_{2}$ will make the system's dynamic performance better, but too much of which will lead to longer adjustment time.

\subsection{Deformation Control}

4.1.1. Attitude Control Law Experiment. Figure 3 shows the attitude step response of the actively deformed aerial robot. It can be seen that the attitude 3-channel controller performs well.

4.1.2. Simulation of Spot Hover during Deformation. For the convenience of observation, the simulation in this paper reverses the value sign of position $z$; that is, the negative sign becomes a positive sign. Set the initial position of the active deformation aerial robot $P_{0}^{E}=[0 ; 0 ; 0]$ and the desired position $P_{d}^{E}=[2 ; 4 ; 6]$. As shown in Figure 4 , the active deformed aerial robot using the ADRC controller designed in this paper can quickly reach and hover at the desired position stably. Deformation is carried out at $10 \mathrm{~s}$ of the simulation. Figures 5 and 6 are the simulation curves of the elongation and folding of a single arm, and Figures 7 and 8 are the simulation curves of the double-arm elongation and folding. Also Figure 9 is the simulation result of the four-arm folding.

It can be seen from Figures 5 and 6 that the active deformation aerial robot can still hover steadily when a single arm is deformed, with the position hardly changing. 
TABLE 2: Simulation parameters of the system.

\begin{tabular}{lcc}
\hline Symbols & Descriptions & Values \\
\hline$m_{\text {body }}$ & Body mass & $31.58 \mathrm{~kg}$ \\
$m_{\text {arm }}$ & Arm mass & $0.43 \mathrm{~kg}$ \\
$m_{\text {rot }}$ & Motor mass & $0.63 \mathrm{~kg}$ \\
$m_{\text {elo }}$ & Mass of telescopic steering gear & $0.31 \mathrm{~kg}$ \\
$g$ & Acceleration of gravity & $9.76 \mathrm{~m} / \mathrm{s}^{2}$ \\
$I_{x x} / I_{y y}$ & $x / y$-axis moment of inertia & $16.38 \mathrm{~kg} \cdot \mathrm{m}^{2}$ \\
$I_{z z}$ & $z$-axis moment of inertia & $30.65 \mathrm{~kg} \cdot \mathrm{m}^{2}$ \\
$k_{f}$ & Lift coefficient & $\mathrm{N} / \mathrm{rad}^{2}$ \\
$k_{m}$ & Antitorque coefficient & $6.33 \times 10^{-5}$ \\
$J_{P}$ & Gyro moment coefficient & $6.1 \times 10^{-7} \mathrm{~N} \cdot \mathrm{mad}^{2}$ \\
$\rho$ & Air density & $5.1 \times 10^{-5} \mathrm{~N} \cdot \mathrm{m} / \mathrm{rad}^{2}$ \\
$S$ & Average windward area & $1.293 \mathrm{~kg} / \mathrm{m}^{3}$ \\
$c$ & Coefficient of air resistance & $0.096 \mathrm{~m} \mathrm{~m}^{2}$ \\
$l_{\text {body }} / h_{\text {body }}$ & Body length $/$ width $/$ height & 2.92 \\
$l_{\text {arm }} / w_{\text {arm }} / h_{\text {arm }}$ & Arm length $/$ width $/$ height & $282 / 73 / 41 \mathrm{~cm}$ \\
$r_{\text {mot }} / h_{\text {mot }}$ & Propeller radius $/$ height & $55 / 13 / 4 \mathrm{~cm}$ \\
$r_{\text {rot }} / h_{\text {rot }}$ & Motor radius $/$ height & $64 / 0.33 \mathrm{~cm}$ \\
$l_{\text {elo }} / w_{\text {elo }} / h_{\text {elo }}$ & Telescopic servo length $/$ width $/$ height & $15 / 3 \mathrm{~cm}$ \\
& $6 / 9.2 / 3.1 \mathrm{~cm}$ \\
\hline
\end{tabular}

TABLE 3: Simulation parameters of active disturbance rejection flight controller.

\begin{tabular}{lcccc}
\hline Parameters & $x / y$ & $z$ & $\phi / \theta$ & $\psi$ \\
\hline TD $-\mathrm{r}$ & 0.98 & 21 & 5.81 & 0.42 \\
TD $-\mathrm{h}$ & 0.001 & 0.001 & 0.001 & 5.1 \\
$\mathrm{TD}-\delta$ & 0.002 & 0.004 & 0.004 & 0.002 \\
$\mathrm{ESO}-\beta_{01}$ & 100 & 1000 & 1000 & 100 \\
$\mathrm{ESO}-\beta_{02}$ & 120 & 17380 & 17380 & 350 \\
$\mathrm{ESO}-\beta_{03}$ & 400 & 133820 & 133820 & 710 \\
$\mathrm{ESO}-b$ & 1.4 & 0.98 & 1.84 & 0.98 \\
$\mathrm{NLSEF}-h$ & 1 & - & 1 & 1 \\
NLSEF $-c$ & 1.128 & - & 7 & 7 \\
NLSEF $-\mathrm{r}$ & 2.11 & - & 4 & 4 \\
NLSEF $-k_{1}$ & - & 45.9 & - & - \\
NLSEF $-k_{2}$ & - & 25 & - & - \\
\hline
\end{tabular}

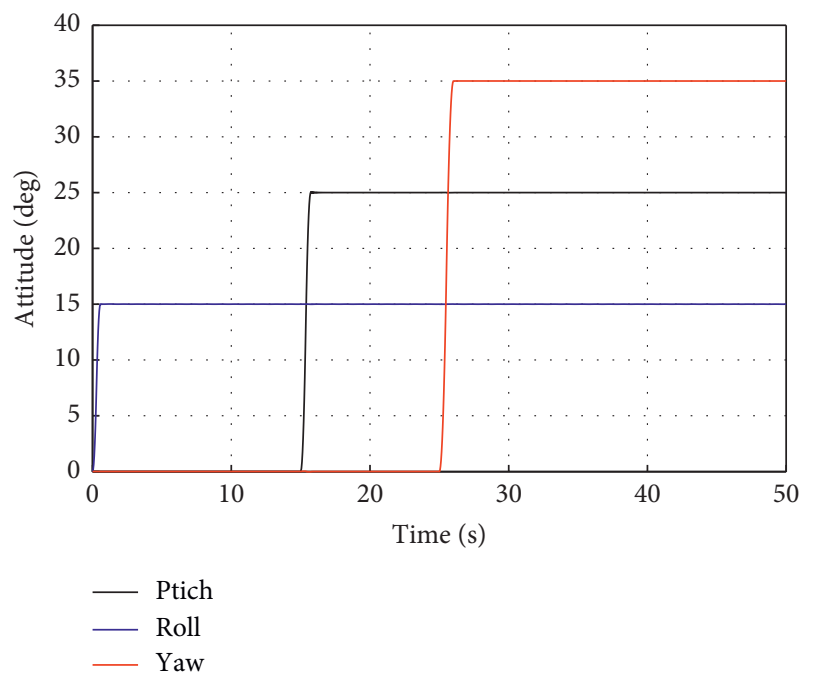

FIGURE 3: Curve of attitude response.
Its attitude is less affected by deformation and the error convergence quickly under the action of the ADRC controller. Conclusion could be made that the control effect is very satisfactory. According to Figures 7 and 8, when the two arms of a deformable robot are deformed, the effect is similar to the deformation with a single arm. It can be seen from Figure 9 that when the four arms of the actively deformed aerial robot are deformed with the same speed simultaneously, there is almost no change in the center of gravity and the inertia tensor, and also its position and posture remain unchanged before the deformation.

4.1.3. Trajectory Tracking Deformation Simulation. The trajectory tracking route of the active deforming aerial robot is designed, and deform is set to happen at $10 \mathrm{~s}$ of tracking. As shown in Figure 10, the deformation method is selected to extend and fold arm 1. Figure 11 shows the trajectory tracking curve of the actively deformed aerial robot, and Figure 12 shows the position change curve during the trajectory tracking of the robot.

It can be seen from Figures 11 and 12 that the active deforming aerial robot is deformed during trajectory tracking and can still maintain a good position tracking effect, which verifies the good position control performance of the designed ADRC controller.

4.2. Simulation of Anti-Interference Performance. Any aircraft in actual flight will inevitably be disturbed by the external environment, especially the disturbance generated by the wind field. In this paper, the Dryden model [31] is used to simulate atmospheric turbulence by shaping and filtering standard Gaussian white noise to obtain white noise. The transfer function of the shaping filter [32] is as follows: 


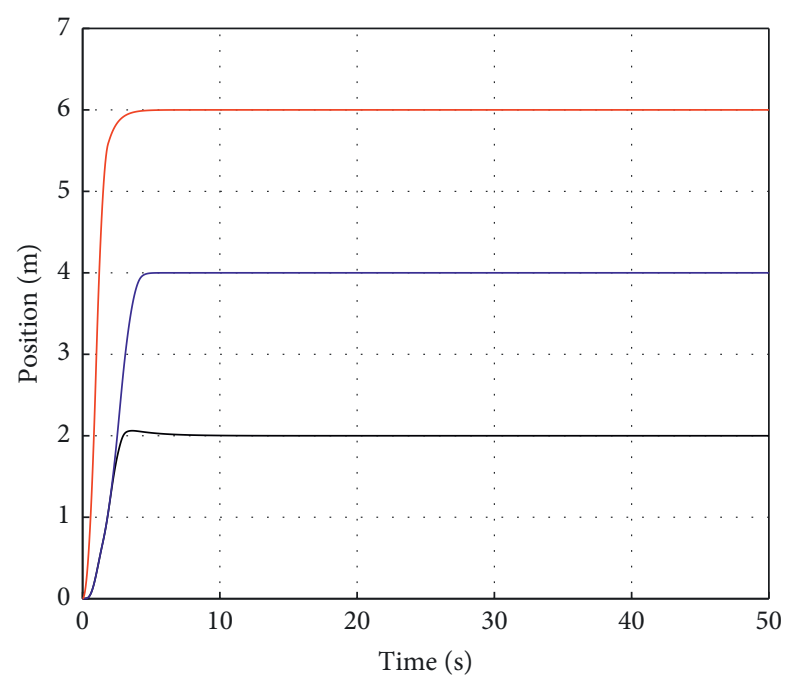

$\left[\begin{array}{l}x \\ y \\ z\end{array}\right.$

FIgURE 4: Curve of position response.

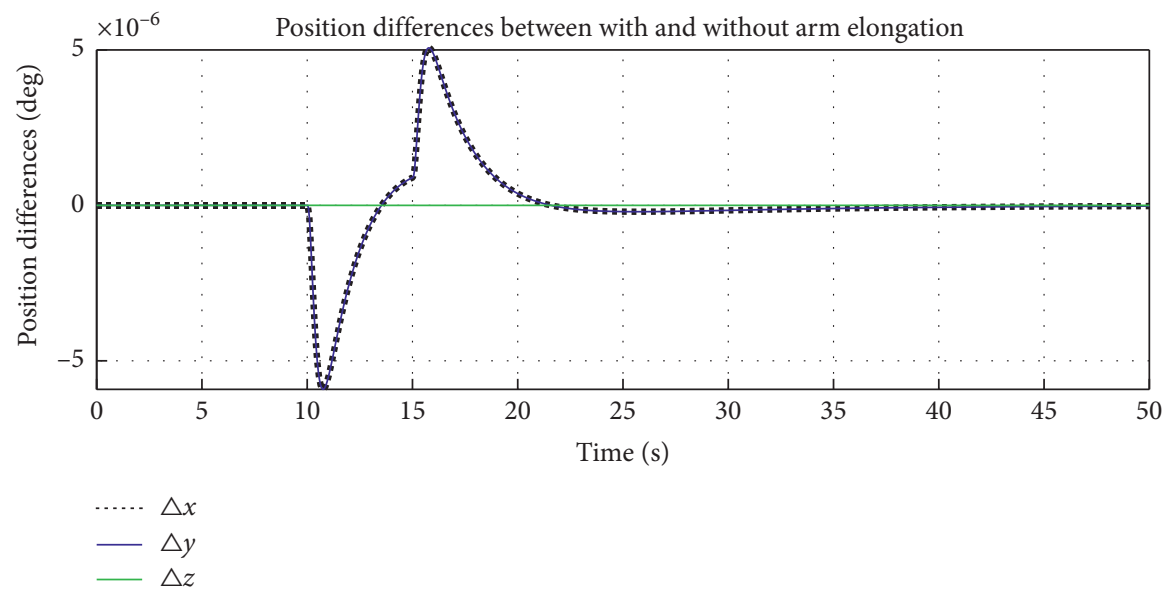

(a)

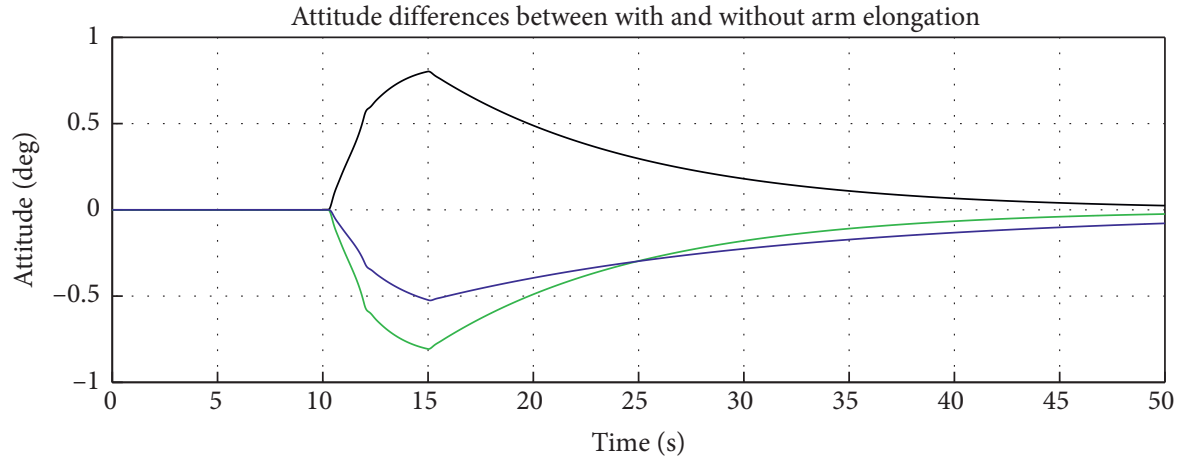

— Ptich

- Roll

_ Yaw

(b)

Figure 5: Curve of single-arm elongation. 


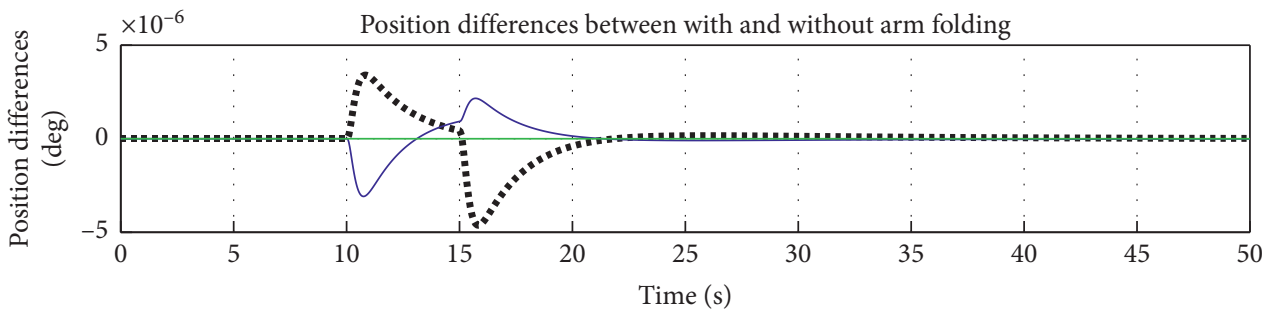

..... $\triangle x$

$-\Delta y$

$-\triangle z$

(a)

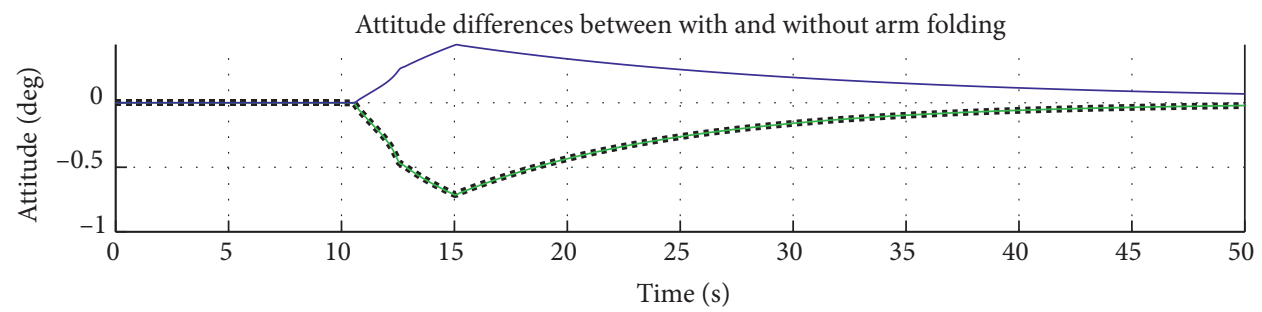

.... Ptich
- Roll
- Yaw

(b)

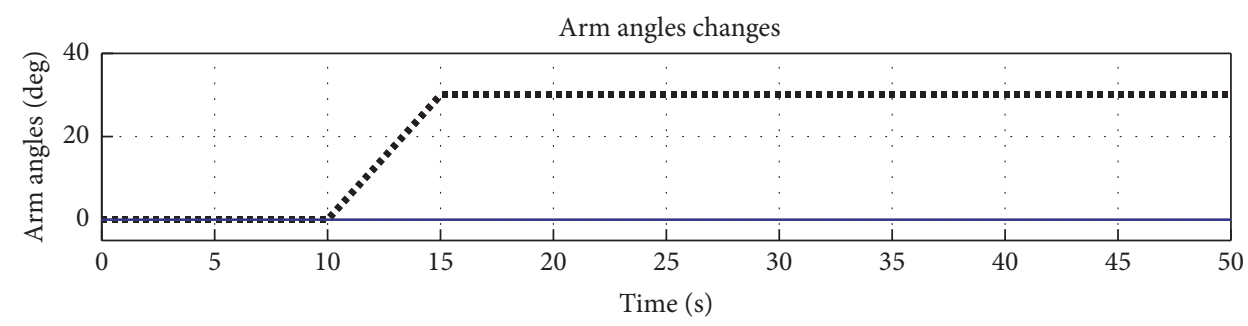

-..... $L 1$

$-L 3$

$-L 2$

$-L 4$

(c)

Figure 6: Curve of single-arm folding.

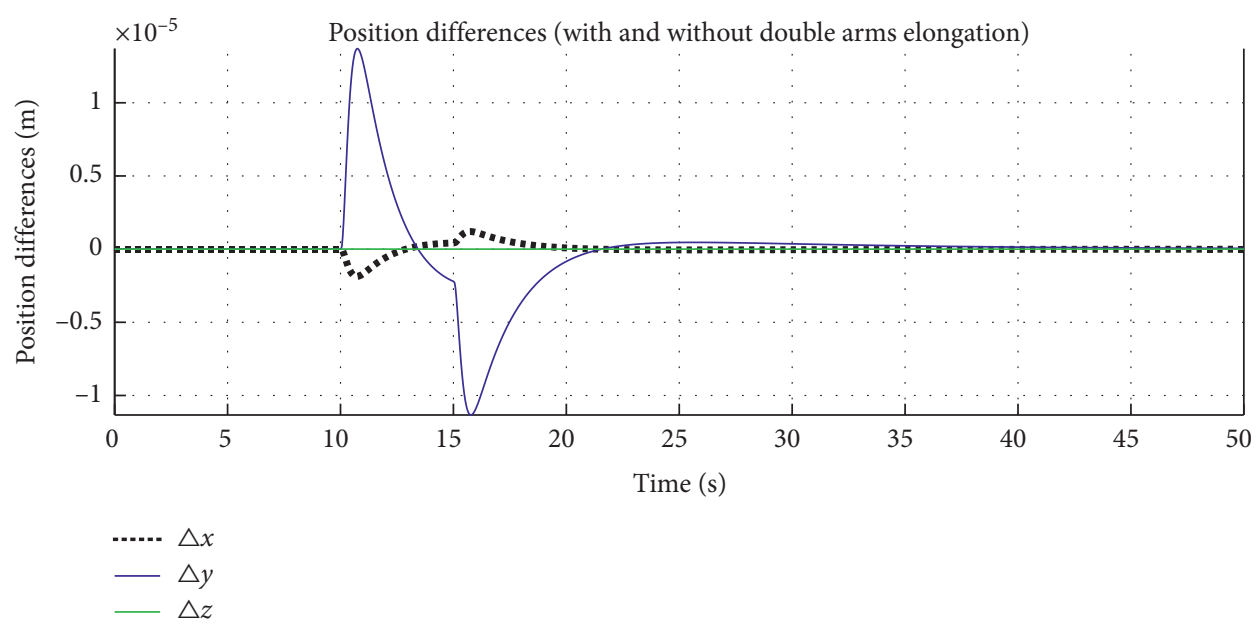

(a)

Figure 7: Continued. 


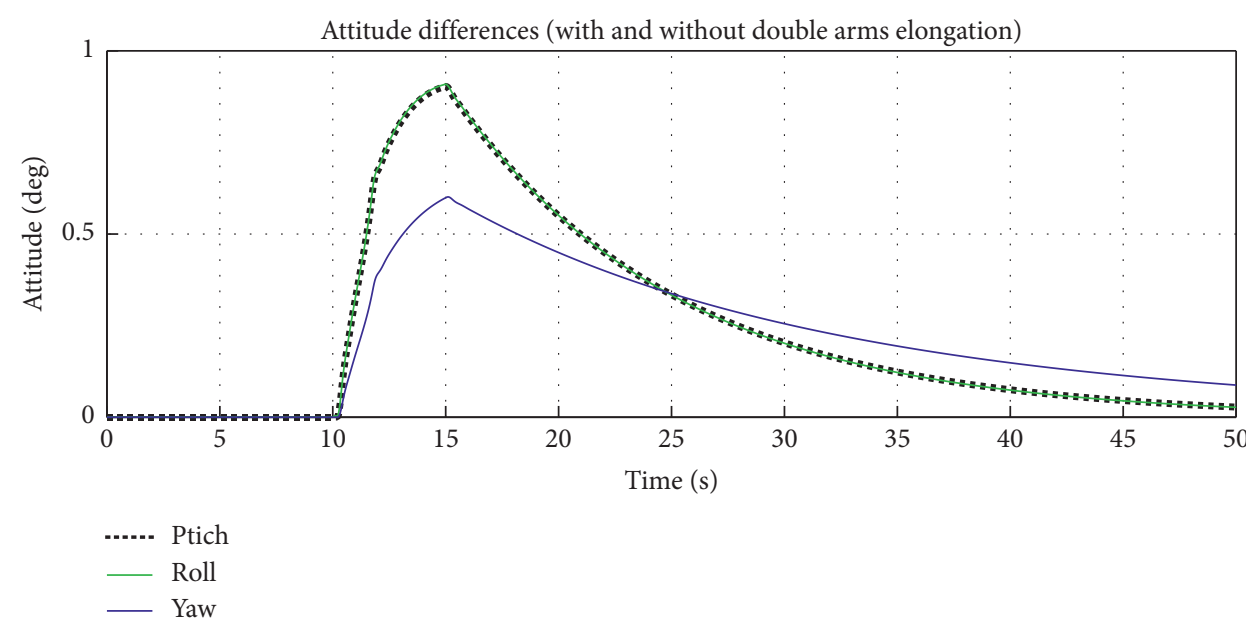

(b)

Figure 7: Curve of double-arm elongation.

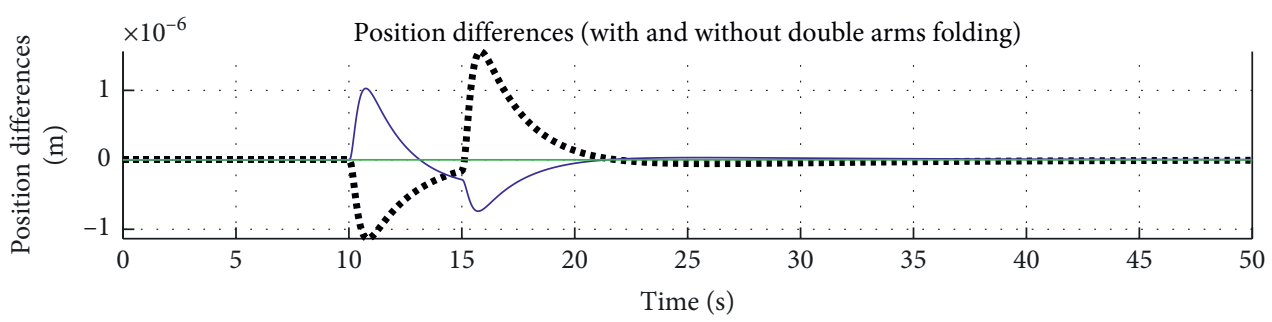

$\begin{aligned} \cdots & \Delta x \\ - & \Delta y \\ - & \Delta z\end{aligned}$

(a)

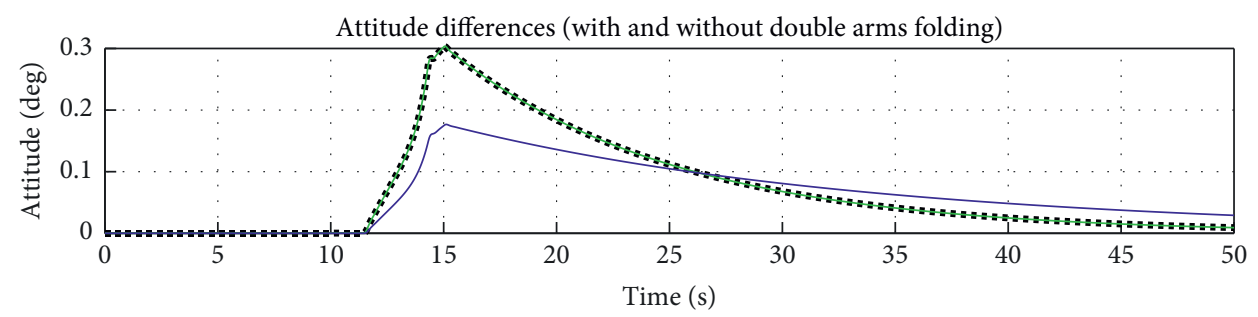

-...-. Ptich

_ Roll

_ Yaw

(b)

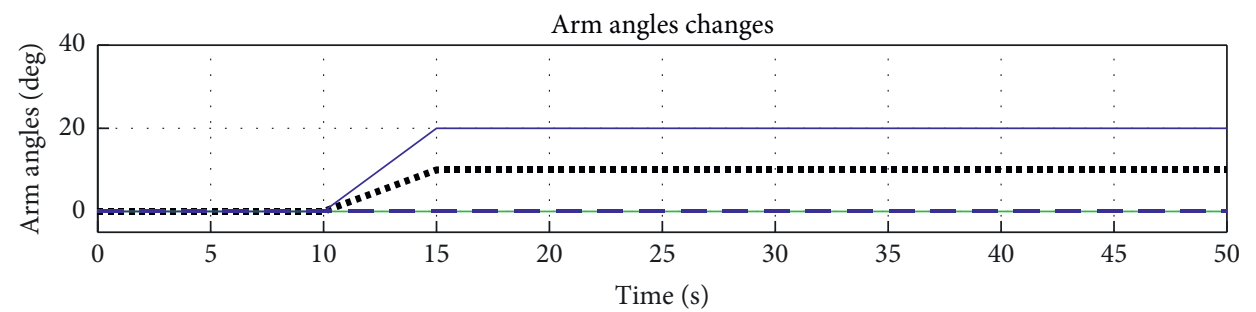

-...- $L 1$

$-L 3$

- L2

-- - L4

(c)

FIgURE 8: Curve of double-arm folding. 


$$
\left\{\begin{array}{l}
G_{u}(s)=\frac{K_{u}}{T_{u} s+1} \\
K_{u}=\sigma_{u} \sqrt{\frac{L_{u}}{\pi v}} \\
T_{u}=\frac{L_{u}}{v}, \\
G_{v}(s)=\frac{K_{v}}{T_{v} s+1} \\
K_{v}=\sigma_{v} \sqrt{\frac{L_{v}}{\pi v}} \\
T_{v}=\frac{2 L_{v}}{\sqrt{3} v}, \\
K_{w}=\sigma_{w} \sqrt{\frac{L_{w}}{\pi v}} \\
G_{w}(s)=\frac{2 L_{w}}{\sqrt{3} v} \\
T_{w} s+1
\end{array}\right.
$$

where $L_{u}, L_{v}, L_{w}$ and $\sigma_{u}, \sigma_{v}, \sigma_{w}$ represent turbulence intensity and turbulence scale, respectively. Rotor aircrafts mainly fly at low altitudes and the calculation formulas for turbulence intensity and turbulence scale under this flight condition are as follows:

$$
\left\{\begin{array}{l}
L_{u}=\frac{h}{(0.177+0.000823)^{12}} \\
L_{v}=\frac{L_{u}}{2}, \\
L_{w}=\frac{h}{2}, \\
\sigma_{u}=\frac{(0.177+0.000823)^{0.4}}{\sigma_{w}} \\
\sigma_{v}=\frac{\sigma_{u}}{(0.177+0.000823)^{0.4}} \\
\sigma_{w}=0.1 u_{20},
\end{array}\right.
$$

where $u_{20}$ represents the wind speed at a height of $6.096 \mathrm{~m}$.

The turbulent wind module is built in MATLAB/ Simulink platform, and the wind field effect formed by superimposing $V_{w}^{E}=[-3 ; 3 ; 0]$ continuous wind is shown in Figure 13. This paper compares ADRC controller, PID controller, and LQR controller and tests the antidisturbance performance of the controller by hovering deformation simulation under 4 different wind field conditions. The deformation is added at $10 \mathrm{~s}$, and the deformation is the same as the single-arm folding in the previous section. The PID parameters used for comparison are set by trial and error. The wind field conditions are as follows:

(1) No wind

(2) Turbulent wind and no continuous wind

(3) Turbulent wind and continuous wind $V_{w}^{E}=[-2 ; 2 ; 0]$

(4) Turbulent wind and continuous wind $V_{w}^{E}=[-3 ; 3 ; 0]$

Set the initial height to $z_{0}=0$ and the expected height to $z_{d}=3$. It can be seen from Figure 14 that when there is no wind, the control effects of ADRC controller, PID controller, and LQR controller are not significantly different. When there is turbulent wind, PID controller and LQR controller begin to fluctuate slightly; and when there are turbulent wind and continuous wind, the PID controller and LQR controller fluctuate more and the actual height is slightly higher than the expected height. The LQR controller is affected by the dual effects of deformation and wind disturbance from $10 \mathrm{~s}$. Compared with the PID controller, the altitude fluctuation is greater. When the continuous wind increases, the flying altitude of the actively deformed aerial robot adjusted by the PID controller and the LQR controller is significantly higher than the expected altitude. Under the same wind field, the ADRC controller can stably control the active deformable aerial robot at the desired height, and the control effect is basically the same as that when there is no wind, which verifies the good antidisturbance performance of the ADRC controller.

Since ESO can observe, control, and compensate for the internal and external disturbances of the system, compared with PID controllers and LQR controllers, ADRC controllers can perfectly suppress deformation disturbances and wind disturbances. It can be seen from equation (18) that the crosswind will cause the rotor to produce wind disturbance in the same direction as the original lift, which increases the total lift. Figure 15 shows the disturbance observed by ESO under wind field condition 94). It can be seen that ESO has good disturbance observation performance, and the observed disturbance direction is consistent with the wind disturbance force direction.

Lastly, to check the antidisturbance ability during the pruning operation, a disturbance of constant pruning force acting around the robot axis $Y$ is added during the attitude tracking control. Figure 16 shows the attitude tracking control with disturbances.

In Figure 16, the constant pruning contact force begins to act from time of $3.0 \mathrm{~s}$ to time of $3.6 \mathrm{~s}$, and it is presented that the robot pitch angle has a slight tremor, but the pruning robot attitude is still in control. 


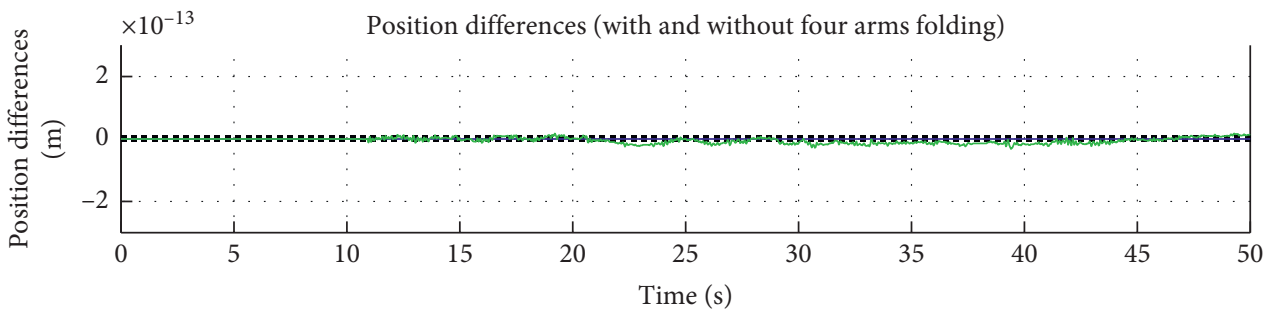

...... $\Delta x$

$-\Delta y$

$-\triangle z$

(a)

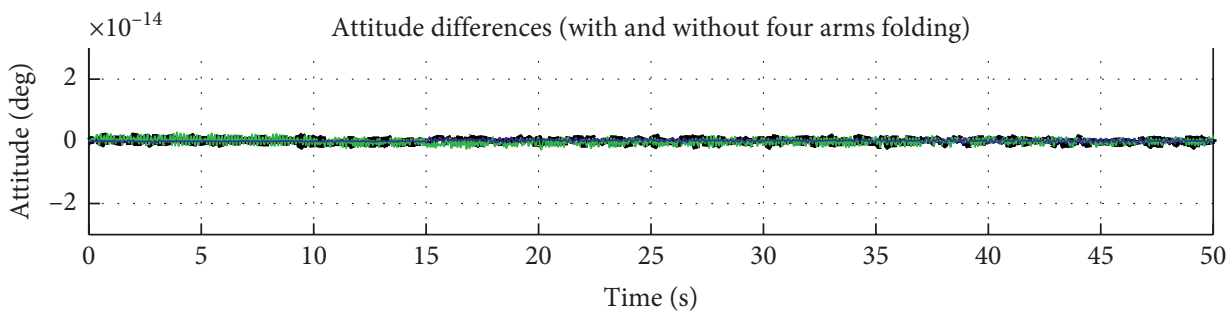

....- $\triangle$ Ptich

$-\triangle$ Roll

$-\triangle$ Yaw

(b)

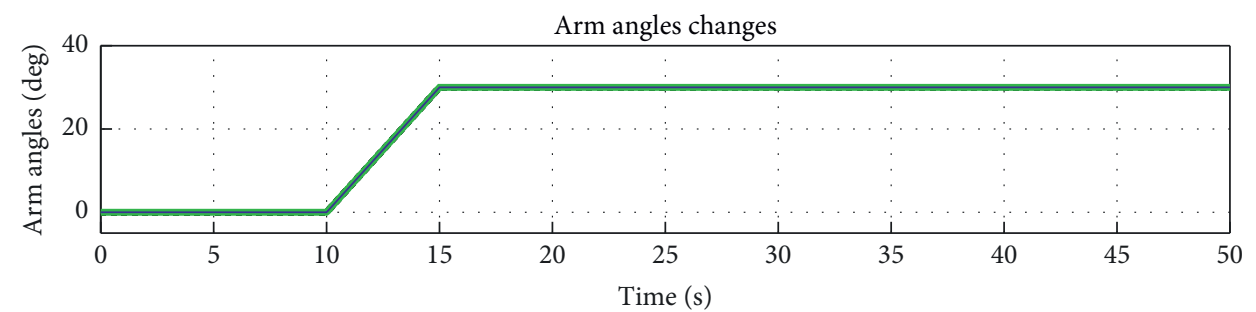

...... $L 1$

$-L 3$

$-L 2$

- - $L 4$

(c)

Figure 9: Curve of four-arm folding.

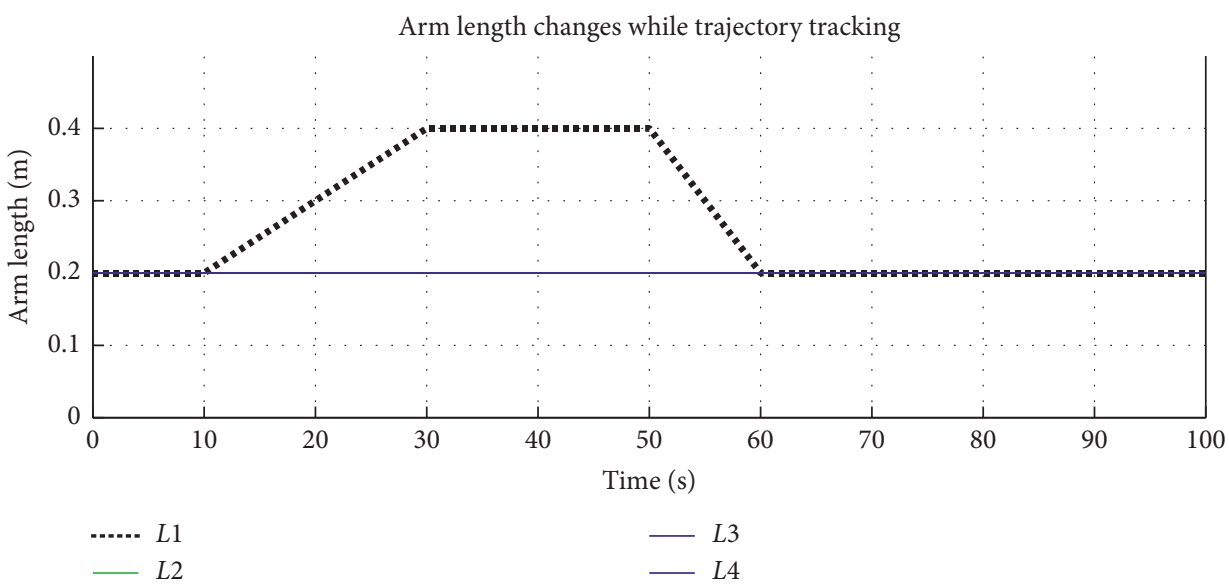

(a)

FIgUre 10: Continued. 


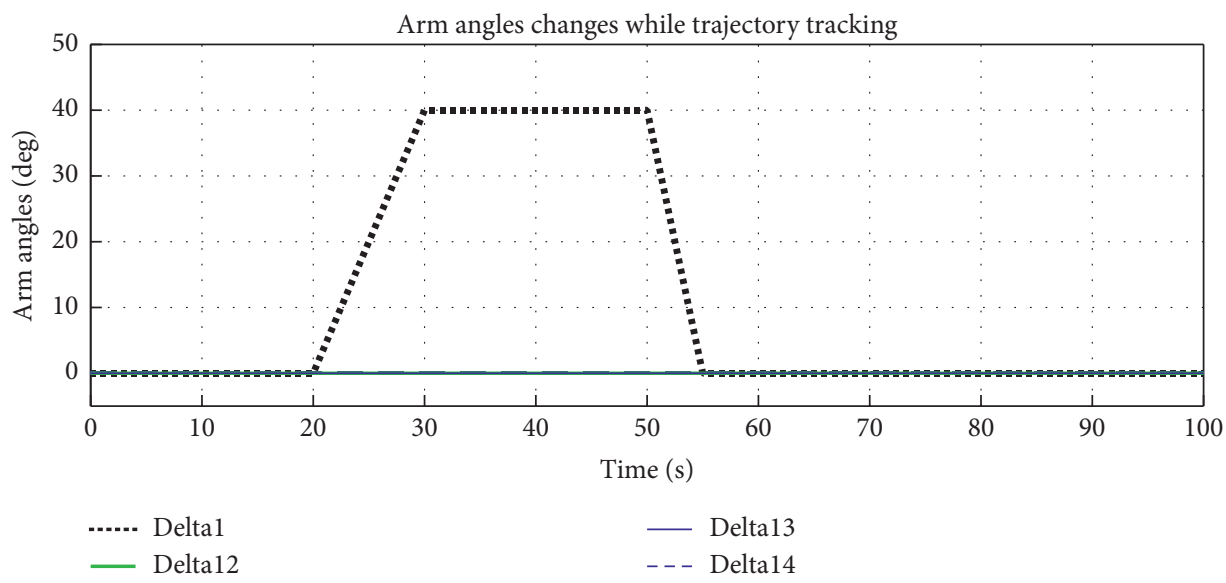

(b)

Figure 10: Change curve of arm length and angle.

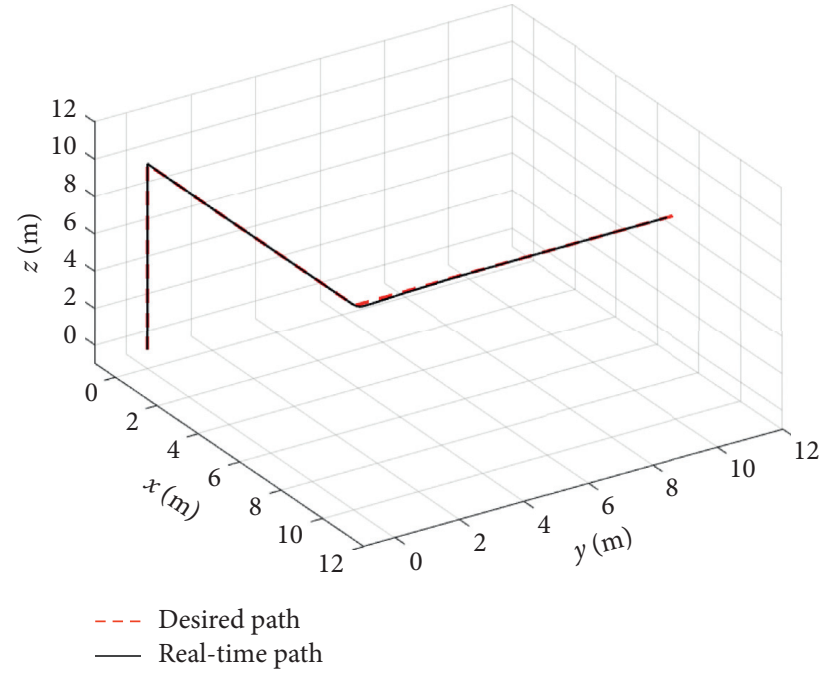

FIGURE 11: Curve of trajectory tracking.

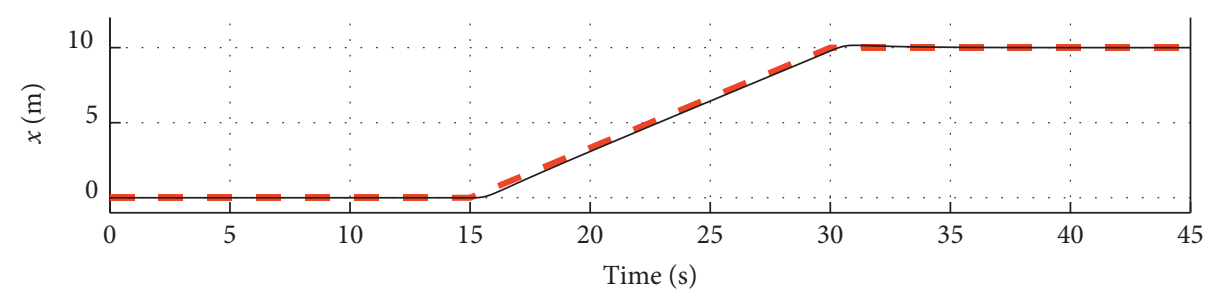

-- Desired path $(x)$
— Real-time path $(x)$

(a)

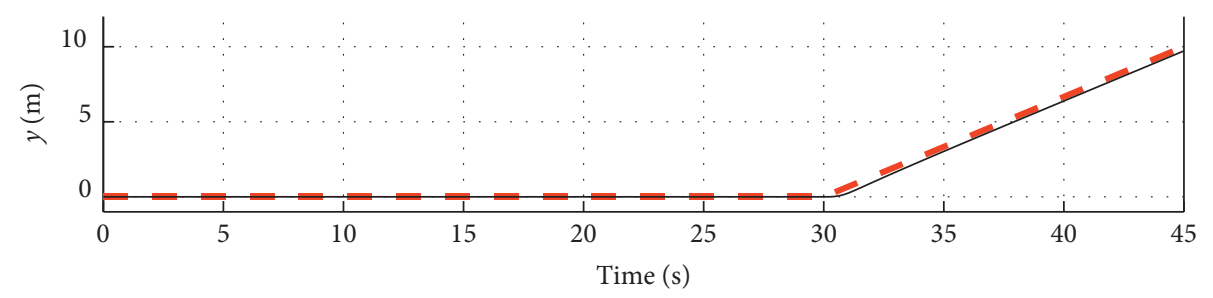

- - - Desired path $(y)$

— Real-time path $(y)$

(b)

FIgURE 12: Continued. 


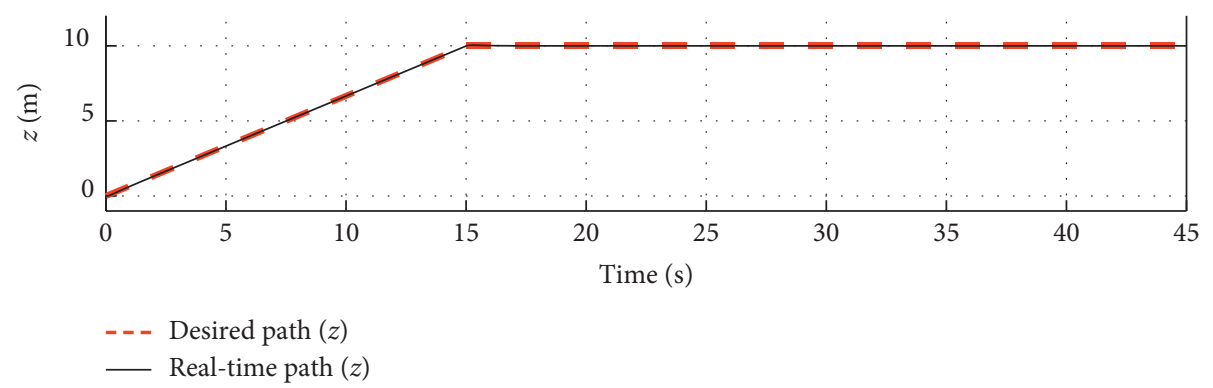

(c)

FIGURE 12: Curve of changing position.

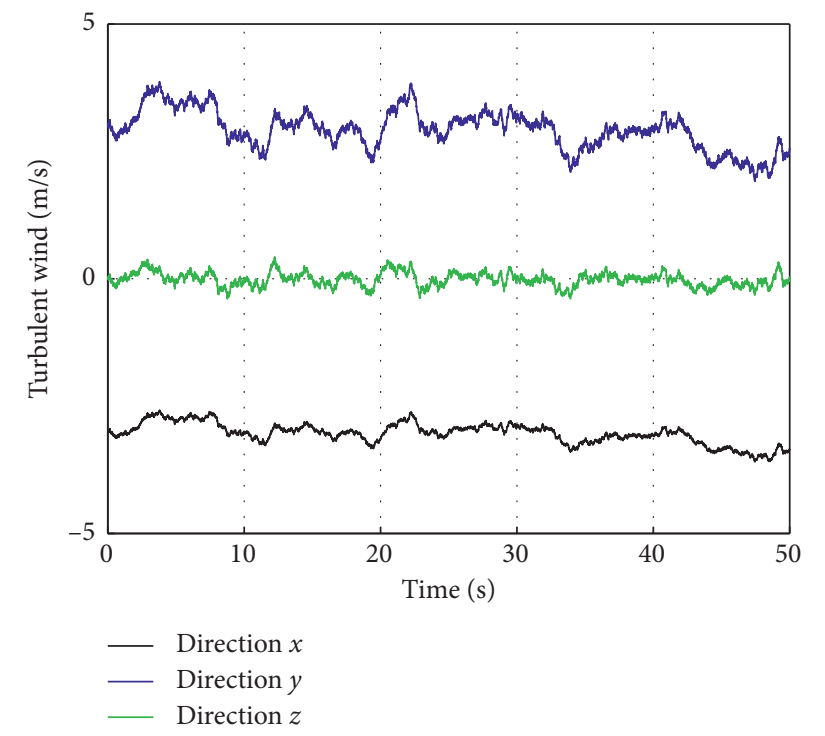

Figure 13: Turbulent flow field.

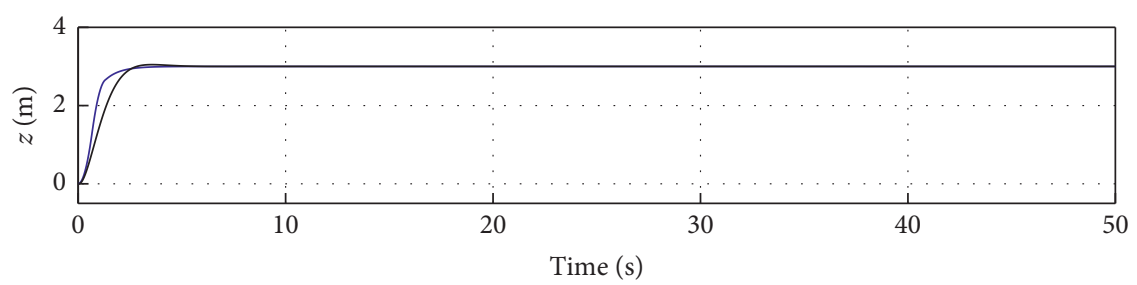

- ADRC (no wind)

— PID (no wind)

(a)

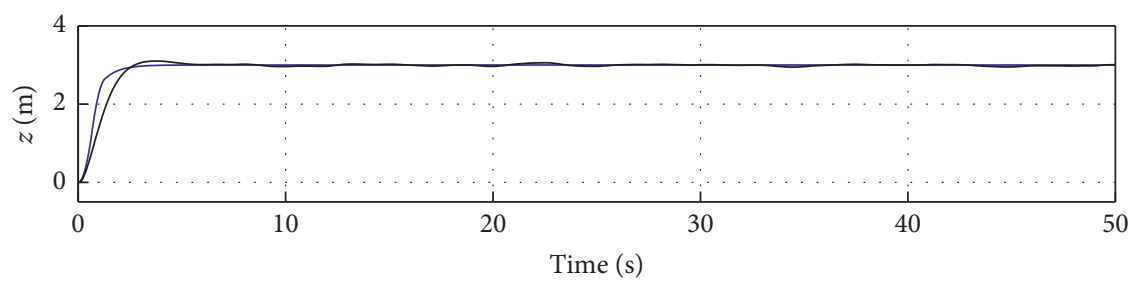

— ADRC (only turbulence)

— PID (only turbulence)

(b)

Figure 14: Continued. 


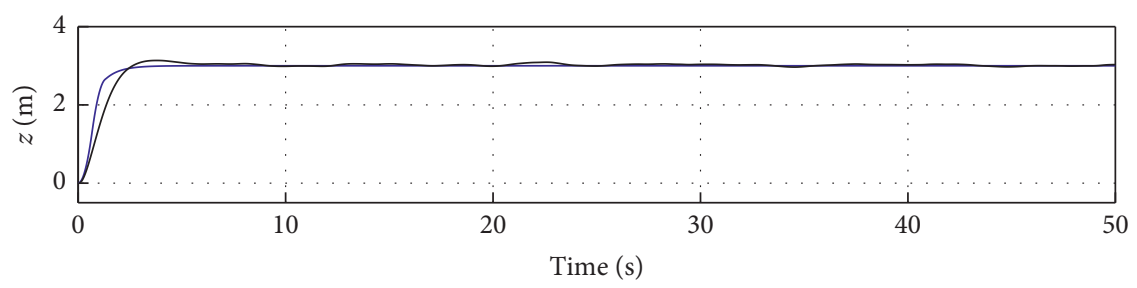

$-\operatorname{ADRC}(1,-1)$

- PID $(1,-1)$

(c)

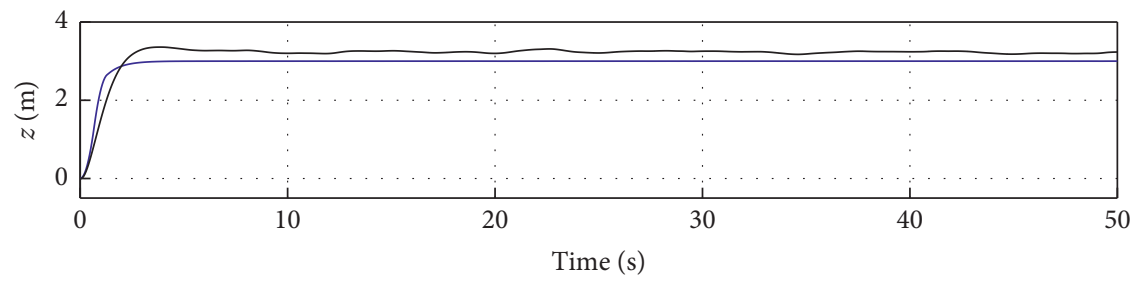

- $\operatorname{ADRC}(3,-3)$

$-\operatorname{PID}(3,-3)$

(d)

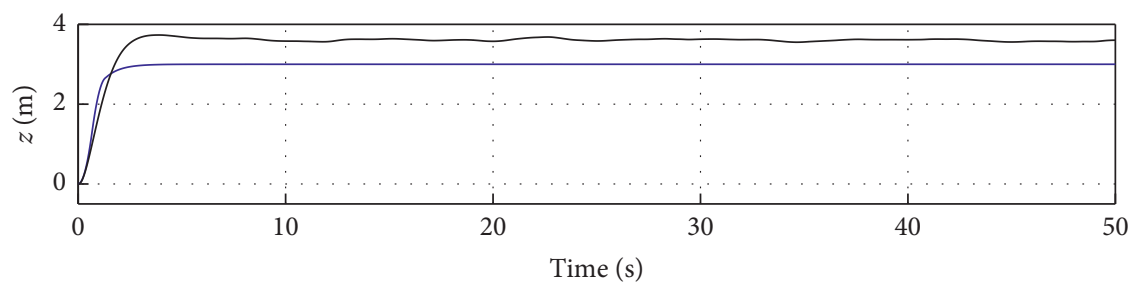

$\operatorname{ADRC}(5,-5)$

- PID $(5,-5)$

(e)

FIGURE 14: Curve of $z$ under wind and without wind.

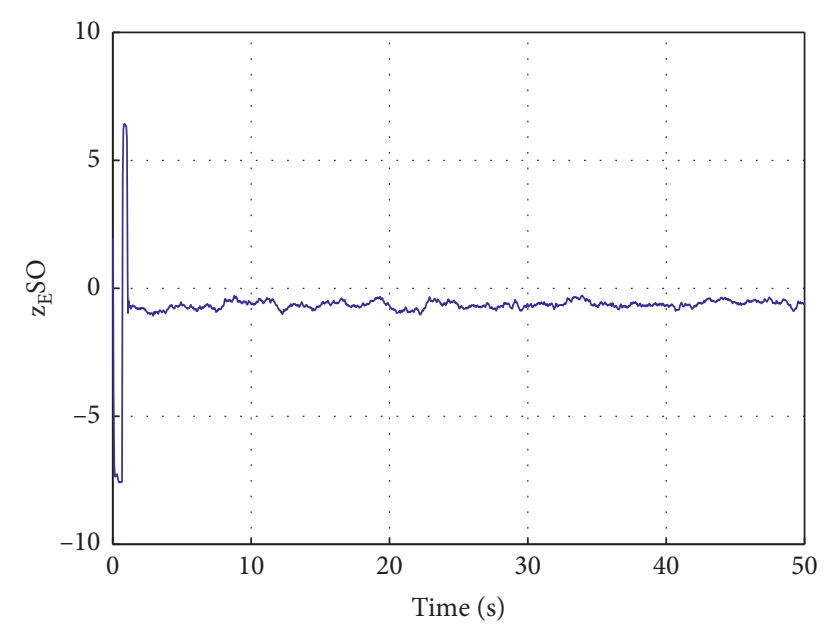

Figure 15: Disturbance estimation of $z$ channel under wind condition.

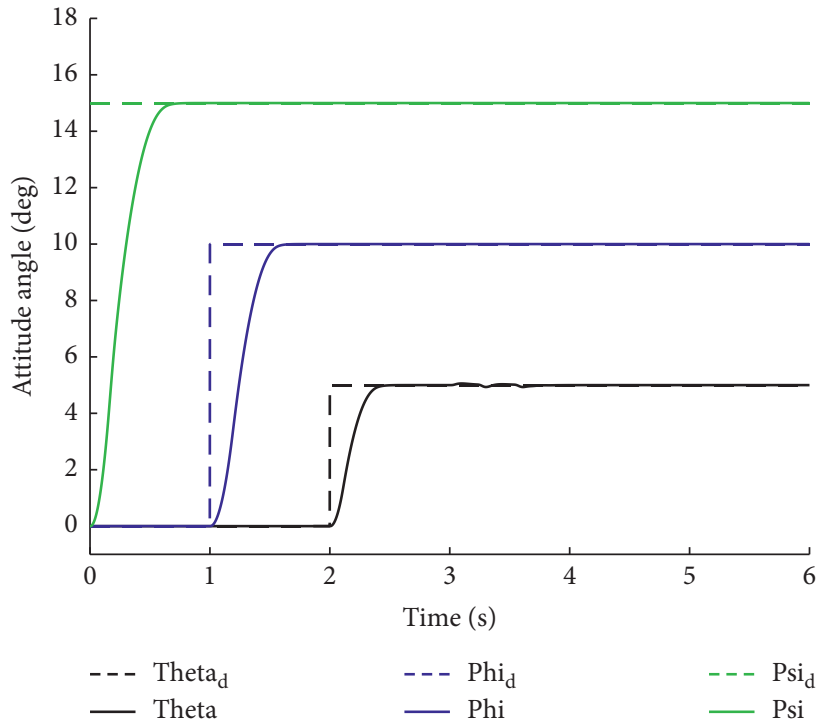

FIgURE 16: Tracking control with pruning disturbances. 


\section{Conclusion}

In order to improve the environmental adaptability of treespruning aerial robots, this paper proposes an active deformable aerial robot with telescopic and foldable arms and designs a control distribution scheme and ADRC controller for it. The simulation results show that the active deformation aerial robot based on the ADRC controller has excellent attitude control ability; not only can it deform well during flight but also it effectively estimates and compensates for the disturbance and turbulent wind disturbance generated during deformation with better performance.

\section{Data Availability}

All data used to support the findings of this study are included within the article.

\section{Conflicts of Interest}

The authors declare that there are no conflicts of interest.

\section{Acknowledgments}

This work was supported by the Guizhou Provincial Science and Technology Projects (Guizhou-Sci-Co-Supp [2020] 2Y044), the Science and Technology Projects of China Southern Power Grid Co., Ltd. (066600KK52170074), the Key Laboratory Projects of Aeronautical Science Foundation of China (201928052006 and 20162852031), the project funded by the Priority Academic Program Development of Jiangsu Higher Education Institutions, and the Research Innovation Program for Postgraduates of Universities in Jiangsu Province (KYLX16_0380).

\section{References}

[1] R. Li, Y. Huang, and J. Liu, "Effective approach to calculate analysis window in infinite discrete gabor transform," Complexity, vol. 2018, 10 pages, 2018.

[2] S. Hayat, S. Wang, and J.-B. Liu, "Valency-based topological descriptors of chemical networks and their applications," Applied Mathematical Modelling, vol. 60, pp. 164-178, 2018.

[3] H. Wang, Y. Huang, and C. Xu, "ADRC methodology for a quadrotor UAV transporting hanged payload," in Proceedings of the IEEE International Conference on Information and Automation (ICIA), pp. 1641-1646, Ningbo, China, August 2016.

[4] X. Ding, P. Guo, K. Xu et al., "A review of aerial manipulation of small-scale rotorcraft unmanned robotic systems," Chinese Journal of Aeronautics, vol. 32, no. 1, pp. 200-214, 2018.

[5] Y. Alothman, M. Guo, and D. Gu, "Using iterative LQR to control two quadrotors transporting a cable-suspended load," The International Federation of Automatic Control (IFAC)PapersOnline.vol. 50, no. 1, pp. 4342-4349, 2017.

[6] D. Brescianini and R. D'Andrea, "Design, modeling and control of an omni-directional aerial vehicle," in Proceedings of the IEEE International Conference on Robotics and Automation (ICRA), pp. 3261-3266, IEEE, Stockholm, Sweden, May 2016.

[7] M. Kamel, S. Verling, O. Elkhatib et al., "The voliro omniorientational hexacopter: an agile and maneuverable tiltable-rotor aerial vehicle," IEEE Robotics \& Automation Magazine, vol. 25, no. 4, pp. 34-44, 2018.

[8] E. Mahmoud, M. Elshafei, A. Saif, and F. Mohamed, "Modeling and simulation of quadrotor UAV with tilting rotors," International Journal of Control, Automation and Systems, vol. 14, no. 4, pp. 1047-1155, 2016.

[9] M. Ryll, H. H. Bulthoff, and P. R. Giordano, "A novel overactuated quadrotor unmanned aerial vehicle: modeling, control, and experimental validation," IEEE Transactions on Control Systems Technology, vol. 23, no. 2, pp. 540-556, 2015.

[10] N. Zhao, Y. Luo, H. Deng et al., "The deformable quadrotor: design, kinematics and dynamics characterization, and flight performance validation," in Proceedings of the IEEE/RSJ International Conference on Intelligent Robots and Systems (IROS), pp. 2391-2396, IEEE, Vancouver, Canada, September 2017.

[11] M. Zhao, K. Kawasaki, X. Chen et al., "Whole-body aerial manipulation by transformable multirotor with two-dimensional multilinks," in Proceedings of the IEEE International Conference on Robotics and Automation (ICRA), pp. 5175-5182, IEEE, Singapore, June 2017.

[12] M. Zhao, T. Anzai, F. Shi, X. Chen, K. Okada, and M. Inaba, "Design, modeling, and control of an aerial robot dragon: a dual-rotor-embedded multilink robot with the ability of multi-degree-of-freedom aerial transformation," IEEE Robotics and Automation Letters, vol. 3, no. 2, pp. 1176-1183, 2018.

[13] B. Li, H. Wang, X. Liu et al., "Design of the scalable folding quad rotor aircraft," Mechanical Research \& Application, vol. 02, pp. 121-124, 2015.

[14] H. E. N. G. Shuai, Design and Implementation of Micro Deformable Quadrotor, Harbin Institute of Technology, Harbin, China, 2016.

[15] D. Falanga, K. Kleber, S. Mintchev et al., "The foldable drone: a morphing quadrotor that can squeeze and fly," IEEE Robotics and Automation Letters, vol. 4, no. 2, pp. 209-216, 2018.

[16] J. Han, "Auto disturbances rejection control technique," Frontier Science, vol. 1, pp. 25-32, 2007.

[17] J. Han, "Auto-disturbances-rejection controller and its applications," Control and Decision, vol. 13, no. 1, pp. 19-23, 1998.

[18] J. Han, "From PID technique to active disturbances rejection control technique," Control Engineering of China, vol. 3, no. 4, pp. 17-22, 2002.

[19] W. Chenlu, C. Zengqiang, S. Qinglin et al., "Chinese control and decision conference (CCDC)-design of PID and ADRC based quadrotor helicopter control system," in Proceedings of the Control \& decision conference. IEEE, pp. 5860-5865, Las Vegas, NV, USA, December 2016.

[20] L. Yang, W. Zhang, and D. Huang, "Robust trajectory tracking for quadrotor aircraft based on ADRC attitude decoupling control," Journal of Beijing University of Aeronautics and Astronautics, vol. 41, no. 6, pp. 1026-1033, 2015.

[21] K. Chang, Y. Xia, K. Huang, and D. Ma, "Obstacle avoidance and active disturbance rejection control for a quadrotor," Neurocomputing, vol. 190, no. 19, pp. 60-69, 2016.

[22] Q. I. Hao-Ran and Q. I. Xiao-Hui, "Research on quadrotor UAV based on linear active disturbance rejection control technique under wind-disturbance," Flight Dynamics, vol. 36, no. 2, pp. 21-25, 2018.

[23] Q. Zhao, H. Wu, and J. Zhu, "Study on the recursive algorithms of mass center for the multi-joint arm boom lift robot," Robot, vol. 28, no. 1, pp. 50-53, 2006. 
[24] J. Zou and J. Zhang, "Parallel calculation of inertial matrix of manipulator," Robot, vol. 19, no. 5, pp. 344-349, 1997.

[25] Y. Li and Y. Wang, "Coordinate transformation of robot's rotational inertia tensor," Robot, vol. 14, no. 2, pp. 31-35, 1992.

[26] K. T. Nguyen, E. Bulka, and N. Meyer, "Quadrotor control in a wind field," in Proceedings of the International Conference on Unmanned Aircraft Systems, pp. 320-328, IEEE, Athens, Greece, September 2015.

[27] K. Lu, Z. Yang, Q. Zhang et al., "An active disturbance rejection flight control method for thrust-vectored quadrotor with tiltable rotor," Control Theory \& Applications, vol. 37, no. 6, pp. 1377-1387, 2020.

[28] A. T. Conlisk, "Modern helicopter rotor aerodynamics," Progress in Aerospace Sciences, vol. 37, no. 5, pp. 419-476, 2001.

[29] Y. He, Y. Chen, and M. Zhou, "Modeling and control of a quadrotor helicopter under impact of wind disturbance," Journal of Chinese Inertial Technology, vol. 21, no. 5, pp. 70-76, 2013.

[30] L. S. Atheer, M. Moghavvemi, H. A. F. Mohamed et al., "Modelling and PID controller design for a quadrotor unmanned air vehicle," in Proceedings of the IEEE International Conference on Automation: IEEE Computer Society, pp. 1-5, Denver, CO, USA, 2010.

[31] T. R. Beal, "Digital simulation of atmospheric turbulence for Dryden and von Karman models," Journal of Guidance, Control, and Dynamics, vol. 16, no. 1, pp. 132-138, 1993.

[32] Y. Qu, Z. Xing, D. Yuan et al., "Wind field estimation based on position and attitude information of quadrotor in hover," Journal of Northwestern Polytechnical University, vol. 34, no. 4, pp. 684-690, 2016. 